\title{
Survival, reproduction and congestion: the spaceship problem re-examined
}

\author{
Pierre-André Jouvet*and Gregory Ponthiere ${ }^{\dagger}$
}

April 28, 2010

\begin{abstract}
This paper re-examines the spaceship problem, i.e. the design of the optimal population under a fixed living space, by focusing on the dilemma between adding new beings and extending the life of existing beings. For that purpose, we characterize, under time-additive individual welfare depending negatively on population density, the preference ordering of a utilitarian social planner over lifetime-equal histories, i.e. histories with demographic conditions yielding an equal finite number of life-periods (imposed by resources constraints). The analysis of the spaceship problem contradicts widespread beliefs about the populationism of Classical Utilitarianism and the antipopulationism of Average Utilitarianism. We also study the invariance property exhibited by various utilitarian rankings to the total space available and to individual preferences. Finally, we compare histories for a spaceship with a stationary population, and try to accomodate intuitions about posterity and renewal of populations.

Keywords: environmental congestion, fertility, longevity, population ethics, utilitarianism, renewal.

JEL codes: D63, Q56, Q57.
\end{abstract}

*EconomiX, University Paris-Ouest, Nanterre - La Défense, and CORE. Address: University Paris-Ouest, 200, avenue de la République, building K and G, 92001 Nanterre, France.

$\dagger$ Paris School of Economics and Ecole Normale Supérieure, Paris [corresponding author]. Address: Ecole Normale Supérieure, Department of Social Sciences, boulevard Jourdan, 48, building B, 75014 Paris, France. E-mail: gregory.ponthiere@ens.fr Telephone: 0033-1-43-1362-04. Fax: 0033-1-43-13-62-22. 


\section{Introduction}

Given that the Earth is of finite size - and, thus, can be compared, following Boulding (1966), to a 'spaceship' - the question of the optimal population size can be formulated as the 'spaceship problem': are there too few or, on the contrary, too many beings living on our bounded, resources-finite, spaceship? That question, which is today at the centre of sustainable development debates, is actually an old issue, to which various answers were given over time.

While Mercantilism was, during the 16th and 17th centuries, promoting a population as large as possible by means of various policies, it should not be deduced from this that the spaceship constraint was ignored by Mercantilist thought. ${ }^{1}$ Actually, it is quite the opposite: a standard Mercantilist argument was that the finiteness of the living space, if coupled with a large population, would favour migrations, and, hence, the colonization of other territories.

The Italian philosopher Giovanni Botero (1588) is generally regarded as one of the first thinkers who argued against the Mercantilist populationism, and asked the question of the optimal population size. Botero argued that men have a tendency to multiply themselves as much as nature allows. However, natural resources are limited, so that, according to Botero, one cannot escape the following adjustments: either people will modify their behaviours, or there will be some adjustment in numbers through famines, diseases or wars.

Within economic thought, Richard Cantillon (1755) provided another early study of the spaceship problem, by highlighting the existence of a quantity of life versus quality of life trade-off, which restrains feasible population sizes. According to Cantillon, Man's subsistence requires living space, whose amount depends on lifestyles, so that an arbitrage is to be made between the quantity and quality of life. ${ }^{2}$ While Cantillon did not solve that trade-off, he showed that, contrary to Mercantilists' beliefs, more people is not always better. ${ }^{3}$

Another contribution to the spaceship problem was made by Thomas Malthus (1798), who argued that the population size is necessarily limited within some boundaries. A population would follow a geometric progression if left unchecked (i.e. in the absence of resources constraints), but the production of means of subsistence follows, at best, an arithmetical progression (because of the finiteness of land), so that the population must, at some point, be 'checked', either by a positive check (deaths) or by a preventive check (fewer children). ${ }^{4}$

Given that Botero, Cantillon and Malthus's positive theories, by underlining the constraints imposed by the finiteness of land, only restrict the set of feasible populations, these leave open the normative question of the optimal population.

That issue was widely studied within utilitarianism. While Jeremy Bentham's (1789) Classical Utilitarianism recommends a number of people producing 'the greatest happiness of the greatest number', one may also, following

\footnotetext{
${ }^{1}$ On Mercantilism and population, see Schumpeter (1954, 1, pp. 352-356).

${ }^{2}$ See Cantillon's comparison of peasants living modestly in the South of France with grownup bourgeois living in abundance (1755, p. 25).

${ }^{3}$ See Cantillon (1755, I, 15, p. 30):

'It is also a question outside of my subject whether it is better to have a great multitude of inhabitants, poor and badly provided, than a small number, much more at their ease: a million who consume the produce of 6 acres per head or 4 million who live on the product of an acre and a half.'

${ }^{4}$ In later writings, Malthus (1830) will add a third type of check: the moral restraint.
} 
Sidgwick's (1874) insights, consider the maximization not of total welfare, but of average welfare, which differs from Benthamite utilitarianism in different numbers choices. However, as shown by Parfit (1984), those two criteria of population ethics are unsatisfactory. Classical Utilitarianism suffers from the Repugnant Conclusion, whereas Average Utilitarianism faces the Mere Addition Paradox. ${ }^{5}$ Hence, other population ethics criteria were developed, such as Critical-Level Utilitarianism (Blackorby and Donaldson 1984) and NumberDampened Utilitarianism (Ng 1986). But although (partly) immunized against Parfit's criticisms, these suffer from other weaknesses. Imposing a low critical level still implies the Repugnant Conclusion, while a high critical level leads to what Arrhenius and Bykvist (1995) call the Sadistic Conclusion. ${ }^{6}$ Moreover, Number-Dampened Utilitarianism might lack intuitive support.

Although much has been written on the optimal population of the Earth spaceship, the specificity of this paper with respect to the existing literature is precisely to consider the spaceship problem on the basis of new fundamentals. Actually, instead of studying the optimal population size without specifying the structure of the population, we propose to re-examine that issue under a more precise specification of the populations at stake. For that purpose, we will distinguish here between the two ends of the demographic 'chain': births and deaths. The intuition is the following. As stressed by Broome (2004), the issue of the optimal number of people is not independent from the issue of the optimal longevity. True, making a person exist and making an existing person live more are, from a moral point of view, two distinct things, but these tend both to influence the number of people at a given point in time, so that the two ends of the demographic chain are relevant for the spaceship problem.

Therefore, we propose a re-examination, from a utilitarian perspective, of the spaceship problem, in which both reproduction and survival are explicitly modelized. The question raised is the following. Suppose that a population, which cares about living a long life in a large living space, has to share a space of finite size. How does the optimal population look in terms of fertility rate and longevity? To answer that question, we will make some significant simplifications, so that the present study should only be regarded as a first step towards a characterization of the solution of the spaceship problem.

First, in order to formalize the longevity versus fertility trade-offs, we will characterize the preferences of a social planner over lifetime-equal histories, defined by demographic conditions (i.e. initial population, fertility and survival rates) yielding an equal finite number of life-periods. ${ }^{7}$ The intuition behind assuming a finite length of history is that some constraints, such as a given stock of non-renewable resources, may tend to limit the total number of periods that can be collectively lived on the spaceship. However, in the last part of the paper we relax that assumption and focus on a spaceship with a stationary

\footnotetext{
${ }^{5}$ The Repugnant Conclusion is defined as follows: for any large population, there is a much larger population with a much lower welfare per head, but which is regarded as better. The Mere Addition Paradox consists of regarding as undesirable the addition of people with a welfare slightly lower than the initial average welfare, even if the welfare of the initial population is unaffected. Average Utilitarianism violates the Mere Addition Principle, according to which adding persons with a positive welfare should not make things worse ceteris paribus.

${ }^{6}$ The Sadistic Conclusion consists of preferring a small population with an extremely low utility to a very large population just below the critical level (see Bykvist 2007).

${ }^{7}$ Reasoning under a fixed total number of life-periods is an analytically convenient way to account for longevity versus fertility trade-offs, to be refined further in future works.
} 
population. That latter case coincides with an infinite history.

Moreover, this paper will not consider here the question of the optimal diversity of the population to be put on the spaceship, contrary to the literature dedicated to the Noah's Ark Problem (see Weitzman 1998). On the contrary, we will, in this paper, focus on the characterization of the population that yields the maximum welfare under a uniform composition of that population.

Furthermore, in our model, the numbers's pressure comes only from population density, which reduces welfare through a pure congestion effect (see Cramer et al 2004). Thus, we will not study here the production of goods, which, since Malthus's work, has occupied a central place in the spaceship problem. Given the observed growth of production per head despite the large population growth, production limitations due to the finiteness of land do not seem likely. ${ }^{8}$

Finally, given the intuitive weaknesses faced by various criteria of population ethics (see Blackorby et al 2005), it makes sense to re-examine the spaceship problem on the basis of several ethical criteria. However, due to space constraints, we shall here focus only on some widely used normative criteria, such as Classical, Average, Critical-Level and Number-Dampened Utilitarianisms, and leave other criteria aside, which is also a significant simplification.

The paper is organized as follows. Section 2 introduces our framework. Section 3 studies a simplified spaceship problem, in which the population cannot reproduce itself, and characterizes the ordering of lifetime-equal histories under various utilitarian criteria. Section 4 explores the more general problem where the population can reproduce itself. Section 5 considers a stationary spaceship (i.e. with a constant asymptotic population). Section 6 concludes.

\section{The spaceship problem: general setting}

The spaceship problem consists of the choice of a population to be put on a spaceship, that is, a place with a finite surface for life. By the terms 'choosing a population', we mean the choice of not only an initial group of people (i.e. the 'pioneers') to be put on the spaceship, but, also, the choice of survival conditions for them, as individual longevity is, given the finiteness of the spaceship, affected by the size of the group of pioneers. Congestion is not neutral for longevity, because overpopulation can prevent the access to vital resources (through depletion, pollution or other phenomena). Thus, depending on the more or less large initial size of the group of pioneers, each pioneer will have, given the finiteness of the spaceship, a more or less short life.

Examples of spaceship problems are numerous. One can consider the selection of an optimal number of astronauts to be sent into space on a rocket. In that case, there will be, in general, no opportunity for astronauts to reproduce themselves in the spaceship, so that survival conditions depend exclusively on the size of the initial group. Another example of a spaceship problem consists of the selection of a population to live on a planet. In that case, survival conditions depend on the size of the initial group, but there is a difference with respect

\footnotetext{
${ }^{8}$ Actually, as Heilig (1994) argues in the light of (mistaken) past studies on the maximum carrying capacity of the Earth in terms of population, the finiteness of space does not seem to be a problem for production, so that we can abstract from that constraint. One may go further, and argue, following Kremer (1994), that population pressure is good for production, as technological progress is proportional to the population size.
} 
to the rocket case: the general living conditions on the planet may allow the reproduction of persons, so that survival conditions depend also on the fertility behaviour, which may cause shorter lives through environmental congestion.

\subsection{Assumptions}

Before examining those various kinds of spaceship problems in more detail, let us first formally introduce the general spaceship problem and the assumptions that we will make throughout this paper.

- Assumption A1 A place of finite surface $Q$ is available for the life of a population.

- Assumption A2 Only living persons occupy a part of the space $Q$.

Moreover, all living persons at a particular point in time $t=0,1,2, \ldots, \infty$, whatever their age is, are assumed to occupy an equal amount of space, defined as the total space divided by the number of people alive at that time. ${ }^{9}$

- Assumption A3 Each person alive at time $t$ enjoys an equal share of the total available space: $q_{t}=\frac{Q}{L_{t}}$, where $L_{t}$ denotes the population size at time $t$, whereas $q_{t}$ denotes the space available per person.

Here are the assumptions we make on individual welfare.

- Assumption A4 At each period, the temporal welfare of a person who is dead equals 0 :

$$
u_{t}=0 \quad \forall t \geq T
$$

where $T$ denotes the date of death of the person.

- Assumption A5 At each period, the temporal welfare of a person who is alive depends on the space available per person, according to the function:

$$
u_{t}=u\left(q_{t}\right)=\left(q_{t}\right)^{\sigma}+\alpha \forall t<T
$$

where $0<\sigma<1$ and $\alpha$ is the intercept of the temporal utility function.

Assumption A4 is a standard normalization. ${ }^{10}$ Assumption A5 is a simple way to capture the disutility from environmental congestion due to overpopulation. According to A5, adding one person to a spaceship of finite size necessarily decreases the welfare of every individual on the spaceship. ${ }^{11}$ Note that, if $\alpha \geq 0$, being alive is always better than being dead, whatever the level of $q_{t}$ is. On the contrary, if $\alpha<0$, there exists a level of $q_{t}$ that makes a person indifferent between, on the one hand, one life-period with that space, and, on the other hand, being dead during that period.

\footnotetext{
${ }^{9}$ Thus we will abstract here from problems of intragenerational distribution of space.

${ }^{10} \mathrm{~A} 4$ allows us also to avoid difficulties associated with the possibility of infinite utility, which would occur under a non-zero utility of death, as dead persons remain dead infinitely.

${ }^{11}$ In reality, it is only below some threshold for $q_{t}$ that adding one person reduces the welfare of every inhabitant. Thus A5 is a strong simplification made for analytical convenience.
} 
- Assumption A6 The lifetime welfare $w$ of a person is the sum of the utilities associated to each life-period:

$$
w=\sum_{t=0}^{T} u\left(q_{t}\right)
$$

Assumption A6 is, although largely questioned by Bommier (2006), still a standard one, due to its simplicity. Let us now turn to the characterization of the demography.

- Assumption A7 The initial population of the spaceship, denoted by $L$, is finite and strictly positive $(L>0)$.

- Assumption A8 At each passage of time, a fraction $S$ of the population alive at a period survives to the next period $(0<S<1)$.

Assumption A8 amounts to assuming that the strength of mortality is constant over the lifecycle: at each passage of time, a constant fraction of a cohort disappears from the spaceship. Assumption A8 is made to restrict the longevity dimension to a single parameter, $S$.

- Assumption A9 A person surviving to the second period of life will give birth to $n$ children at the beginning of that second period $(n \geq 0)$.

Assumption A9 amounts to supposing that the 'intensity of desires' (in Malthus's words) or the need for a dynasty is constant across people and times. ${ }^{12}$ This allows us to confine fertility concerns to a single parameter, $n .{ }^{13}$

\subsection{Problem setting}

In order to study how a social planner solves the spaceship problem, defined as the choice of an initial number of persons $L$, a proportion of survivors $S$, and a fertility rate $n$ under the space constraint, we assume that the social planner has to choose among a set of histories, defined as follows.

- Definition D1 A history is a triplet $\{L, S, n\}$, where $L$ is the size of the population at time $0(L>0), S$ is the proportion of survivors from a period $t$ to a period $t+1(0<S<1)$, and $n$ is the fertility rate.

In order to describe the planner's solution, we shall concentrate on a particular subset of the set of histories: lifetime-equal histories, defined as follows. ${ }^{14}$

\footnotetext{
${ }^{12}$ A9 amounts to assuming that all births are located, for a given person, during, and only during, the second period of his life, and, more exactly, at the beginning of that second period. For instance, the $S^{2} L$ members of cohort 0 who are alive in their third period of life do not give birth to any offspring in period 2 and beyond, but only at the beginning of period 1 , and the utility of their children is counted for the whole period during which they are born.

${ }^{13}$ Note that this model does not distinguish between males and females, so that $n$, the fertility rate per person, concerns all people, and not only women as in real life. A fertility rate of 2 children per women would correspond to $n=1$ in this model.

${ }^{14}$ To be precise, lifetime-equal histories mean total lifetime-equal histories, in the sense that it is the total sum of the lifetimes of all agents that is equal across histories. In the rest of the paper, we will use the term 'lifetime-equal histories' for short.
} 
- Definition D2 Two histories $\{L, S, n\}$ and $\{\tilde{L}, \tilde{S}, \tilde{n}\}$ are lifetime-equal if and only if these exhibit an equal total number of life-periods.

Note that the total number of life-periods for the first cohort (i.e. the pioneers), denoted by $P_{0}$, is ${ }^{15}$

$$
P_{0}=L+S L+S^{2} L+\ldots=L \frac{1}{1-S}
$$

The total number of life-periods for the second cohort, denoted by $P_{1}$, is

$$
P_{1}=S L n+S^{2} L n+S^{3} L n+\ldots=L S n \frac{1}{1-S}
$$

One can then define the total number of life-periods for all other cohorts, denoted by $P_{2}, P_{3}, \ldots$ etc. The total number of life-periods for a cohort $j$ is

$$
P_{j}=L n^{j} S^{j} \frac{1}{1-S}
$$

Hence the total number of life-periods, denoted by $P$, is

$$
P=\sum_{t=0}^{\infty} P_{t}=L \frac{1}{1-S}\left(1+n S+n^{2} S^{2}+\ldots\right)
$$

Note that $P$ is infinite if $n S \geq 1$. However, for $n S<1$, we have

$$
P=\frac{L}{(1-S)(1-n S)}
$$

That ratio can be decomposed in two factors: $\frac{1}{1-S}$ is the average longevity for any person, that is, the inverse of the strength of mortality, whereas $\frac{L}{1-n S}$ is the total number of persons ever born: it is the product of the initial population size $L$ and the strength of fertility $\frac{1}{1-n S}$ (i.e. the average number of descendants for each person in the initial population). Thus the product of those two factors $\frac{1}{1-S}$ and $\frac{L}{1-n S}$ yields the total number of life-periods $P$.

Note that the total number of life-periods is increasing in the initial population size $L$ (i.e. $\frac{\partial P}{\partial L}>0$ ), increasing in the survival rate $S$ (i.e. $\frac{\partial P}{\partial S}>0$ ), and also increasing in the fertility rate $n\left(\frac{\partial P}{\partial n}>0\right)$.

In order to remain within the field of finite ethics, we will, in Sections 3 and 4 , concentrate on the case where $n S<1$, guaranteeing a finite total number of life-periods, that is, a mankind history of finite length. ${ }^{16}$

- Assumption A10 Only a finite number of life-periods will be lived on the spaceship: $n S<1$.

Assumption A10 can be regarded as a constraint resulting from the finiteness of, let us say, the stock of some non-renewable natural resources present on

\footnotetext{
${ }^{15}$ In this paper, we mean, by a 'life-period', a 'period lived by someone'. For instance, if two persons live one period, we say that the total number of life-periods equals two.

${ }^{16}$ See Section 5 for a study of the case where $n S=1$.
} 
the spaceship. ${ }^{17}$ Under A10, we focus on lifetime-equal histories $\{L, S, n\}$ and $\{\tilde{L}, \tilde{S}, \tilde{n}\}$, which, by definition, are such that:

$$
\frac{L}{(1-S)(1-n S)}=\frac{\tilde{L}}{(1-\tilde{S})(1-\tilde{n} \tilde{S})}
$$

For a given number of pioneers (i.e. $L=\tilde{L}$ ), an equal number of life-periods (i.e. $P=\tilde{P}$ ) means that if one lifetime-equal history exhibits a higher fertility $n$ than another one, it must also exhibit a lower survival rate $S$.

Whereas the comparison of lifetime-equal histories $\{L, S, n\}$ and $\{\tilde{L}, \tilde{S}, \tilde{n}\}$ can take various forms, we will start with the simple case where there is no possibility for the initial population to reproduce itself (i.e. $n=\tilde{n}=0$ ). Then, in a second stage, we will consider the case where reproduction exists, so that new people can appear at each point in time $(n>0$ and $n S<1)$. Finally, we will turn to the case where $n S=1$, which yields an infinite number of periods lived by a population, whose size converges asymptotically towards some constant.

\section{The spaceship problem (1): no reproduction}

This section examines a simplified version of the spaceship problem, in which the population cannot reproduce itself. That subcase of the spaceship problem will be referred to as the spaceship problem without reproduction. The absence of reproduction amounts to assuming the following.

- Assumption A11 The fertility rate $n$ is equal to 0 .

Under A11, all new people are concentrated on the first period, $t=0$, and form the initial population $L$ of finite size. The total number of periods $P$ lived by a population of initial size $L$ is:

$$
P=L+S L+S^{2} L+\ldots=L \frac{1}{1-S}
$$

Thus, if $S$ tends towards $0, P$ tends towards $L$ : the number of life-periods equals the number of persons. However, if $S$ tends towards $1, P$ tends towards $+\infty$ : as there is no death, the number of life-periods is infinite. ${ }^{18}$

Hence, in the spaceship problem without reproduction, comparing two lifetimeequal histories $\{L, S, 0\}$ and $\{\tilde{L}, \tilde{S}, 0\}$ amounts to comparing two histories for which:

\footnotetext{
${ }^{17}$ Note that assuming $n S<1$ yields a total population at time $t$ that converges asymptotically towards 0 . Indeed, writing $L_{t}$ the number of people alive at time $t$, we have

$$
L_{\infty}=\lim _{t \rightarrow \infty} L_{t}=L S^{\infty}+(L S n) S^{\infty-1}+\left(L S^{2} n^{2}\right) S^{\infty-2}+\ldots+L\left(S^{\infty} n^{\infty}\right) S n=0
$$

as $S<1$. Thus, our spaceship will, under $n S<1$, end up empty in the long-run. But this asymptotic emptyness does not prevent a temporary growth of the spaceship population: the only thing that it prevents is a permanent growth or a stationary positive size.

${ }^{18}$ It follows from the previous formulae that, if $S$ tends to 1 , the space available per person $q_{t}$ is constant over time and equal to $Q / L$, as: $\lim _{S \rightarrow 1} q_{t}=\lim _{S \rightarrow 1} \frac{Q}{S^{t} L}=\frac{Q}{L}$. In that case, the population that is alive initially will remain forever in the spaceship, and will enjoy the same space conditions during their whole life. On the contrary, if $S$ tends towards $0, q_{t}$ tends to be infinite for any $t>0$, as: $\lim _{S \rightarrow 0} q_{t}=\lim _{S \rightarrow 0} \frac{Q}{S^{t} L}=\frac{Q}{0}=+\infty$. Indeed, as $S$ tends towards 0 , the survivors can enjoy an infinitely large space at each period.
} 


$$
L \frac{1}{1-S}=\tilde{L} \frac{1}{1-\tilde{S}}
$$

which can be rewritten:

$$
\frac{L}{\tilde{L}}=\frac{1-S}{1-\tilde{S}}
$$

that is, there is an equality of the ratios of initial populations (LHS) and strengths of mortality (RHS) for histories $\{L, S, 0\}$ and $\{\tilde{L}, \tilde{S}, 0\}$ having an equal number of life-periods. If one history exhibits a larger initial population than another history with the same total number of life-periods (i.e. $L>\tilde{L}$ ), it must also be characterized by a larger mortality (i.e. $1-S>1-\tilde{S}$ ).

\subsection{The Classical Utilitarian solution}

According to Classical Utilitarianism (CU), as firstly stated by Bentham (1789), all actions - at the individual and institutional levels - should be chosen in such a way as to produce the 'greatest happiness of the greatest number', in conformity with the Principle of Utility. That principle must also govern the choice of histories in general, and, in particular, of lifetime-equal histories. ${ }^{19}$

- Definition D3 A social planner is Classical Utilitarian (CU) if and only if, when facing two histories $\{L, S, n\}$ and $\{\tilde{L}, \tilde{S}, \tilde{n}\}$, he prefers the history yielding the highest total welfare, that is,

$$
\{L, S, n\} \succeq\{\tilde{L}, \tilde{S}, \tilde{n}\} \Longleftrightarrow \sum_{t=0}^{\infty} L_{t} u\left(q_{t}\right) \geq \sum_{t=0}^{\infty} \tilde{L}_{t} u\left(\tilde{q}_{t}\right)
$$

Regarding the preferences $\succeq$ of a CU social planner over lifetime-equal histories, Proposition 1 states a general result: a CU social planner prefers lifetimeequal histories with the smallest population size and the largest life expectancy.

Proposition 1 Assume A1-A11. Consider two lifetime-equal histories $\{L, S, 0\}$ and $\{\tilde{L}, \tilde{S}, 0\}$, with $L>\tilde{L}$ and $S<\tilde{S}$. A CU planner prefers $\{\tilde{L}, \tilde{S}, 0\}$ to $\{L, S, 0\}$, whatever the levels of $Q, \alpha$ and $\sigma$.

Proof. See the Appendix.

The result stated in Proposition 1 is quite surprising, as the existing literature on normative population ethics has, since Parfit's (1984) Repugnant Conclusion, highlighted the tendency of $\mathrm{CU}$ to lead to excessively large populations with low levels of utility per person, a recommendation regarded as 'repugnant' by many theorists. On the contrary, Proposition 1 states that, when facing a choice between lifetime-equal histories, CU selects the history with the smallest population. We are thus far from the populationism associated with CU.

While a proof of Proposition 1 is provided in the Appendix, we can give here some intuition behind CU's preference for small populations. ${ }^{20}$ Actually, if one computes the marginal social welfare gain from raising $L$ while maintaining the

\footnotetext{
${ }^{19}$ Note that the Benthamite objective function does not involve any discounting here, in conformity with Ramsey's (1928) views. Note also that the above formula presents social welfare by aggregating across time periods rather than persons (as usually done), but this is equivalent to aggregating across people, because of the additivity of lifetime welfare.

${ }^{20}$ It should be stressed here that Assumption A5 is a major driving force for that result. If we added, instead of A5, temporal welfare invariant to $q_{t}$ above some threshold of $q_{t}$, then Proposition 1 would no longer be true.
} 
total number of life-periods $L /(1-S)$ constant, one can see that such a marginal social welfare gain is, under $\mathrm{CU}$, necessarily negative. ${ }^{21}$ Thus adding one more being while reducing the expected lifetime of all existing beings in such a way as to maintain total lifetime constant is welfare-reducing under CU.

Note also that Proposition is quite general, as this holds whatever the total space $Q$ is, and for all values of preference parameters $\sigma$ and $\alpha$. That latter point is surprising, as we would expect $\alpha$ to matter for choices between alternatives involving unequal longevities. However, the total number of life-periods is the same in all lifetime-equal histories, and this explains why $\alpha$ is neutral here.

Finally, let us turn to two extreme cases. ${ }^{22}$

- Corollary C1 Under $\sigma=0$, a CU planner is indifferent between any lifetime-equal histories.

When $\sigma=0$, space does not matter at all: only existence matters, and, given the equal number of life-periods in each history, indifference must prevail, under $\mathrm{CU}$, between all lifetime-equal histories.

- Corollary C2 Under $\sigma=1$, a CU planner is indifferent between any lifetime-equal histories.

If temporal welfare is linear in $q_{t}$, the $\mathrm{CU}$ planner is indifferent between all lifetime-equal histories, as the initial population $L$ and the survival rate $S$ affect social welfare in an identical manner in that case.

\subsection{Average Utilitarianism}

Although accepted as an ethical basis in various issues, CU faces intuitive difficulties in the field of population ethics. A major critique of $\mathrm{CU}$ consists of Parfit's (1984) Repugnant Conclusion: for any population with a significant welfare per head, there exists a larger population of individuals with a very low welfare level, but which is ranked as better by CU (the rise in the quantity of lives compensating the fall in the quality of lives). Note, however, that CU does not, in the present context, yield to any populationism. As shown in the previous subsection, CU selects, on the contrary, the lifetime-equal history with the smallest initial population $L$ and the largest survival rate $S$.

${ }^{21}$ Actually, social welfare $W$ under a history $\{L, S, 0\}$ can be written as

$$
W=\sum_{t=0}^{\infty} W_{t}=L^{1-\sigma} Q^{\sigma} \frac{1}{1-S^{1-\sigma}}+\frac{L}{1-S} \alpha
$$

Hence the marginal welfare gain from raising $L$ while reducing $S$ in such a way as to keep $L /(1-S)$ constant is

$$
\frac{\partial W^{c}}{\partial L} \equiv \frac{\partial W}{\partial L}+\frac{\partial W}{\partial S} \frac{\partial S}{\partial L}
$$

where $\frac{\partial W^{c}}{\partial L}$ is the change in welfare induced by a change in $L$ compensated by a change in $S$ to maintain $L /(1-S)$ constant. Clearly, for a total number of periods lived $P$, we have $S=1-\frac{L}{P}$ and thus $\frac{\partial S}{\partial L}=-\frac{1}{P}$. Thus we have, after simplifications:

$$
\frac{\partial W^{c}}{\partial L}=L^{-\sigma} Q^{\sigma}(1-\sigma)\left[\frac{1-S^{-\sigma}}{\left(1-S^{1-\sigma}\right)^{2}}\right]+\frac{1}{1-S} \alpha\left[1-\frac{1}{1-S}\right]<0
$$

That expression is negative, as $0<S<1$.

22 See the Appendix for proofs. 
Having stressed this, it remains that $\mathrm{CU}$ is only one normative criterion among others, which also deserve attention when examining the spaceship problem. Let us thus consider an alternative criterion, Average Utilitarianism (AU). ${ }^{23}$

- Definition D4 A social planner is Average Utilitarian (AU) if and only if, when facing two histories $\{L, S, n\}$ and $\{\tilde{L}, \tilde{S}, \tilde{n}\}$, he prefers the history yielding the highest welfare per existing person, that is,

$$
\begin{gathered}
\{L, S, n\} \succeq\{\tilde{L}, \tilde{S}, \tilde{n}\} \\
\Longleftrightarrow \frac{1-S n}{L} \sum_{t=0}^{\infty} L_{t} u\left(q_{t}\right) \geq \frac{1-\tilde{S} \tilde{n}}{\tilde{L}} \sum_{t=0}^{\infty} \tilde{L}_{t} u\left(\tilde{q}_{t}\right)
\end{gathered}
$$

where $\frac{1-S n}{L}$ is the inverse of the total number of existing persons under an initial population $L$, a survival rate $S$ and a fertility rate $n$.

As stated below, the social ordering over histories under AU may, under some conditions, differ significantly from the one under CU.

Proposition 2 Assume A1-A11. Consider two histories $\{L, S, 0\}$ and $\{\tilde{L}, \tilde{S}, 0\}$, with $L>\tilde{L}$ and $S<\tilde{S}$.

- If $\alpha \geq 0$, an $A U$ planner prefers $\{\tilde{L}, \tilde{S}, 0\}$ to $\{L, S, 0\}$, whatever the levels of $Q$ and $\sigma$. That is, any increase in $S$ is regarded as better, and any decrease in $L$ is regarded as better, independently from any relation between $L$ and $S$.

- If $\alpha<0$, given two lifetime-equal histories $\{L, S, 0\}$ and $\{\tilde{L}, \tilde{S}, 0\}$, with $L>\tilde{L}$ and $S<\tilde{S}$, an AU planner prefers $\{L, S, 0\}$ to $\{\tilde{L}, \tilde{S}, 0\}$ if and only if

$$
\alpha \leq \frac{Q^{\sigma} L^{-\sigma}(1-\tilde{S})(1-S)}{(\tilde{S}-S)(1-S)^{-\sigma}}\left[\frac{(1-S)^{-\sigma}}{1-S^{1-\sigma}}-\frac{(1-\tilde{S})^{-\sigma}}{1-\tilde{S}^{1-\sigma}}\right]
$$

Proof. See the Appendix.

Proposition 2 is quite surprising, as one would expect AU to lead to a preference for histories with a low $L$ and a high $S$ in all cases, exactly as CU. However, as stated in Proposition 2, this is not the case, and thus the standard view of AU as an antipopulationist ethical doctrine needs to be amended. Clearly, the social planner's preferences over lifetime-equal histories depend significantly on the level of the intercept of the temporal utility function $\alpha$.

If $\alpha$ is non-negative, AU recommends the lifetime-equal history with the smallest $L$ and the largest $S$, exactly as CU. Note, however, that, under $\alpha \geq 0$, the preference of the social planner for lifetime-equal histories with few initial persons and large life expectancies is here even stronger than under CU. Indeed, Proposition 2 states that the social planner's preference for histories with low $L$ and high $S$ prevails whatever the relation between $L$ and $S$ is, that is, even if one departs from the comparison of lifetime-equal histories.

Nevertheless, if $\alpha$ is negative, then it is no longer obvious that AU recommends histories with low $L$ and large $S$. Actually, the opposite may be true, provided the intercept $\alpha$ is sufficiently negative. Indeed, for a sufficiently low

\footnotetext{
${ }^{23}$ Regarding the sources of AU, it was argued by Gottlieb (1945) that Mill supported AU implicitly, because he argued in favour of the control of births on the grounds of social welfare (see Mill 1859, p. 242). However, it is Sidgwick (1874, p. 415-416) who first made the explicit distinction between total utility and average utility.
} 
level of $\alpha$, an AU planner prefers $\{L, S, 0\}$ to $\{\tilde{L}, \tilde{S}, 0\}$, contrary to what prevails under CU. Thus, for a very low intercept $\alpha$, the antipopulationist bias of $\mathrm{AU}$ is false, as this leads to a larger population in comparison to CU. ${ }^{24}$ The intuition behind that result is that, under a very negative $\alpha$, it may be optimal, under $\mathrm{AU}$, to opt for a history with a larger $L$ and a lower $S$, as this is a way to 'dilute' the negative utility associated to the fixed time of existence on more people. ${ }^{25}$ A population made of a few long-lived agents who accumulate a large stock of disutility, will, on average, be far worse off than a population made of lots of short-lived agents, who do not have enough lifetime to accumulate a very negative lifetime welfare. This dilution effect does not take place under CU, as there, it is the total stock of disutility - rather than the stock per person - that matters, and this total stock is constant for all lifetime-equal histories.

In sum, the present study contradicts common beliefs. CU is often accused of populationism, and AU is generally suspected of antipopulationism. The examination of the social ordering over lifetime-equal histories invalidates those beliefs. CU recommends lifetime-equal histories with the smallest population, whereas AU may involve the choice of a more populated history than CU.

\subsection{Critical-Level Utilitarianism}

After having studied the solutions to the spaceship problem under $\mathrm{CU}$ and $\mathrm{AU}$, let us now explore the solutions under alternative criteria of population ethics. It was indeed shown in the literature that, whereas AU avoids the Repugnant Conclusion, it suffers from another weakness, which was called the Mere Addition Paradox by Parfit (1984). Clearly, AU regards as undesirable the addition of a group of people with an individual welfare that is slightly lower than the initial average welfare, even if the additional people have a life worth living, and even if the added people do not influence in any way the lives of the initial group. In order to avoid that counter-intuitive result - but without facing the Repugnant Conclusion - one solution proposed by Blackorby and Donaldson (1984) consists of summing, instead of absolute utilities, the contributory values of all lives, defined as the lifetime welfare of existing agents minus the critical level of welfare $\hat{u}(\hat{u} \geq 0)$, which makes life - as a whole - neutral. This yields Critical-Level Utilitarianism (CLU): a rise in the population is desirable if the

${ }^{24}$ To see the intuition behind those results, note that, given $\frac{\partial W^{c}}{\partial L} \equiv \frac{\partial W}{\partial L}+\frac{\partial W}{\partial S} \frac{\partial S}{\partial L}$, we have

$$
\frac{\partial W^{c}}{\partial L}=\frac{(-\sigma) L^{-\sigma-1} Q^{\sigma}}{1-S^{1-\sigma}}+\left[\frac{(1-\sigma) L^{-\sigma} Q^{\sigma} S^{-\sigma}}{\left(1-S^{1-\sigma}\right)^{2}}+\frac{\alpha}{(1-S)^{2}}\right] \frac{\partial S}{\partial L}
$$

If $\alpha \geq 0$, that expression is negative, as the first term is negative. Thus, if $\alpha \geq 0$, the welfare change induced by a rise in $L$ is always negative, even when $\frac{\partial S}{\partial L}=0$. Hence the preference for a low $L$ does not require here $\frac{\partial S}{\partial L}<0$. Inversely, $\frac{\partial W^{c}}{\partial S}$ is always positive, even when $\frac{\partial L}{\partial S}=0$.

But if $\alpha<0$, it is no longer obvious that $\frac{\partial W^{c}}{\partial L}<0$, even when we compare lifetime-equal histories (i.e. $\frac{\partial S}{\partial L}=-\frac{1}{P}$ ). Indeed, we then have

$$
\frac{\partial W^{c}}{\partial L}=-\left(\frac{Q}{L}\right)^{\sigma} \frac{\sigma}{L} \frac{1-S^{-\sigma}}{\left(1-S^{1-\sigma}\right)^{2}}-\frac{1}{L(1-S)} \alpha
$$

Under $\alpha<0$, the first term is negative and the second one is positive, so that the sign of $\frac{\partial W^{c}}{\partial I}$ is ambiguous, and depends on the two lifetime-equal histories under comparison.

${ }^{25}$ This dilution effect presupposes that a fixed time has to be lived (i.e. the total lifetime has to be equal to $P$, and cannot be lower than $P$ ). Of course, a social planner who could opt for a total lifetime lower than $P$ would, under $\alpha<<0$, recommend no lifetime at all. 
welfare of additional people exceeds $\hat{u}$, and undesirable if it is lower than it. ${ }^{26}$

- Definition D5 A social planner is a Critical-Level Utilitarian (CLU) if and only if, when facing two histories $\{L, S, n\}$ and $\{\tilde{L}, \tilde{S}, \tilde{n}\}$, he prefers the history yielding the highest total contributory values of lives, that is,

$$
\begin{gathered}
\{L, S, n\} \succeq\{\tilde{L}, \tilde{S}, \tilde{n}\} \\
\Longleftrightarrow \sum_{t=0}^{\infty} L_{t} u\left(q_{t}\right)-\frac{L}{1-S n} \hat{u} \geq \sum_{t=0}^{\infty} \tilde{L}_{t} u\left(\tilde{q}_{t}\right)-\frac{\tilde{L}}{1-\tilde{S} \tilde{n}} \hat{u}
\end{gathered}
$$

where $\hat{u}$ is the critical welfare level, making a whole life neutral, while $\frac{L}{1-S n}$ is the total number of persons under an initial population $L$, and survival and fertility rates $S$ and $n$.

It is crucial here to distinguish the introduction of that neutral level for existence $\hat{u}$ from the introduction of what Broome (2004) calls a 'neutral level for continuing existence' (i.e. a critical level defined for each period of life). The introduction of such a critical level for continuing existence would not alter the Benthamite condition for $\{L, S, 0\} \succeq\{\tilde{L}, \tilde{S}, 0\}$, unlike what is the case under the introduction of a lifetime critical level $\hat{u}{ }^{27}$

Under the assumption of no reproduction $(n=\tilde{n}=0)$, the preferences of a CLU planner over lifetime-equal histories can be characterized as follows.

Proposition 3 Assume A1-A11. Consider two lifetime-equal histories $\{L, S, 0\}$ and $\{\tilde{L}, \tilde{S}, 0\}$, with $L>\tilde{L}$ and $S<\tilde{S}$. A CLU planner prefers $\{\tilde{L}, \tilde{S}, 0\}$ to $\{L, S, 0\}$, whatever the levels of $Q, \alpha$ and $\sigma$.

Proof. See the Appendix.

Thus, the introduction of a critical level of lifetime utility $\hat{u}$ does not affect the social planner's preferences in comparison with what CU recommends. In each case, the social planner will always prefer lifetime-equal histories with the smallest initial population $L$ and the largest survival rate $S .{ }^{28}$ This independence of the social ordering from the critical lifetime welfare $\hat{u}$ is surprising. But this result is also somewhat reassuring, as recent debates on the selection of an adequate critical utility level seem largely open (see Crisp 2007; Broome 2007). Thus it is good that the solution does not depend on $\hat{u}$.

Moreover, it should also be stressed that the social planner's preferences do not, unlike $\mathrm{AU}$, depend on the intercept of the temporal utility function $\alpha$. The

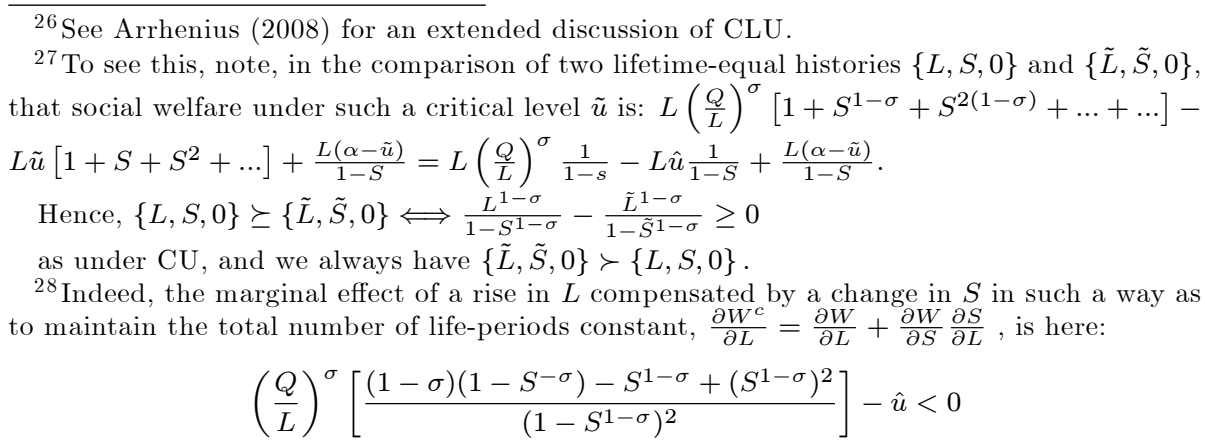

That expression is always negative, whatever the level of $\alpha, \sigma$ and $Q$. 
reason why $\alpha$ is benign here is that, as under CLU, we base the comparison of histories on the total welfare (in net terms) for all life-periods. The total number of life-periods is, by definition, the same for all lifetime-equal histories, and so $\alpha$ has the same effects in all histories under comparison. This was different under AU, as there it was not the total utility of all life-periods that was under comparison, but the utility of all life-periods divided by the number of persons, which varies across lifetime-equal histories, so that $\alpha$ was far from benign.

\subsection{Number-Dampened Utilitarianism}

Although CLU was developed as an alternative to $\mathrm{CU}$ and $\mathrm{AU}$, it is subject to similar problems. If the critical level $\hat{u}$ is too low, the Repugnant Conclusion arises, whereas if it is too high, the Mere Addition Paradox or the Sadistic Conclusion prevails. This observation - and the difficulties to select a single value of $\hat{u}$ - encouraged the development of other criteria. ${ }^{29}$

One of those alternative criteria is Ng's (1986) Number-Dampened Utilitarianism (NDU). ${ }^{30}$ The value function under NDU is equal to the average utility multiplied by a positive-valued function of the population size. When that function is a multiple of the population size, that criterion is equivalent to CU, whereas, if that function is a constant, NDU is equivalent to AU.

Under NDU, the problem of a social planner is to choose $\{L, S, n\}$ in such a way as to maximize the sum of all cohorts's welfares, defined as the product of the average utility in that cohort and a concave transform of its size.

- Definition D6 A social planner is Number-Dampened Utilitarian (NDU) if and only if, when facing two histories $\{L, S, n\}$ and $\{\tilde{L}, \tilde{S}, \tilde{n}\}$, he prefers the history yielding the highest average welfare multiplied by a concave transform of the existing population, that is,

$$
\begin{gathered}
\{L, S, n\} \succeq\{\tilde{L}, \tilde{S}, \tilde{n}\} \\
\Longleftrightarrow\left(\frac{L}{1-S n}\right)^{\gamma}\left[\frac{1-S n}{L} \sum_{t=0}^{\infty} L_{t} u\left(q_{t}\right)\right] \geq\left(\frac{\tilde{L}}{1-\tilde{S} \tilde{n}}\right)^{\gamma}\left[\frac{1-\tilde{S} \tilde{n}}{\tilde{L}} \sum_{t=0}^{\infty} \tilde{L}_{t} u\left(\tilde{q}_{t}\right)\right]
\end{gathered}
$$

where $0 \leq \gamma \leq 1$

Proposition 4 presents the preferences of a NDU social planner over lifetimeequal histories $\{L, S, 0\}$ and $\{\tilde{L}, \tilde{S}, 0\}$.

Proposition 4 Assume A1-A11. Consider two lifetime-equal histories $\{L, S, 0\}$ and $\{\tilde{L}, \tilde{S}, 0\}$, with $L>\tilde{L}$ and $S<\tilde{S}$.

- If $\alpha \geq 0$, a NDU planner prefers $\{\tilde{L}, \tilde{S}, 0\}$ to $\{L, S, 0\}$, whatever the levels of $Q$ and $\sigma$.

- If $\alpha<0$, a NDU planner prefers $\{L, S, 0\}$ to $\{\tilde{L}, \tilde{S}, 0\}$ if and only if

$$
\alpha \leq \frac{Q^{\sigma} L^{-\sigma}(1-\tilde{S})(1-S)}{(1-S)^{\gamma-\sigma}\left[(1-\tilde{S})^{\gamma}(1-S)^{1-\gamma}-(1-\tilde{S})\right]}\left[\frac{(1-S)^{\gamma-\sigma}}{1-S^{1-\sigma}}-\frac{(1-\tilde{S})^{\gamma-\sigma}}{1-\tilde{S}^{1-\sigma}}\right]
$$

\footnotetext{
${ }^{29}$ One solution is the adherence to Critical-Band Utilitarianism (see Blackorby et al 2005), which allows some interval of individual welfare levels between which a life is neutral.

${ }^{30}$ On Number-Dampened Utilitarianism, see also Hurka (1983).
} 
Proof. See the Appendix.

Note that, under $\gamma$ equal to 1 , the condition on $\alpha$ mentioned in the second part of Proposition 4 is never valid, so that we are then back to the ranking of the CU social planner: the lifetime-equal history with the lowest $L$ and the largest $S$ is preferred to other lifetime-equal histories, whatever $\alpha, \sigma$ and $Q$ are. ${ }^{31}$ Moreover, under $\gamma$ equal to 0 , the condition mentioned in the second part of Proposition 4 collapses to the one in Proposition 2, so that the ranking of NDU on lifetime-equal histories is then equivalent to the one under AU.

Let us now contrast the above solution with the one under an alternative generalization of standard utilitarian criteria: CLU. A fundamental difference between NDU and CLU lies in the fact that the ordering of a NDU planner over lifetime-equal histories does not necessarily lead to the choice of the history with the smallest $L$ and the largest $S$, unlike CLU. Such a difference is surprising, as CLU and NDU are generally regarded as common generalizations of CU and AU. The spaceship problem in its simplest form reveals that there is a strong division between, on the one hand, CU and CLU, which both always lead to the selection of the lifetime-equal history with the lowest $L$ and the largest $S$, whatever the intercept of temporal utility $\alpha$ is, and, on the other hand, AU and NDU, for which the solution depends on the level of $\alpha$. For those two criteria, it may be optimal, under a very negative $\alpha$, to opt for a history with an initial population that is larger than the minimum one, in such a way as to dilute the fixed, miserable total lifetime on a larger number of persons. ${ }^{32}$ Such a dilution does not occur under CU and CLU, for which increasing the population size does not bring any improvement (as dilution is here impossible).

\subsection{A synthesis}

Let us now collect our results regarding the social ranking of lifetime-equal histories in a spaceship problem without reproduction. For that purpose, Table 1 shows the sensitivity - or, alternatively, the invariance - of the social ranking of lifetime-equal histories to the structural parameters of the economy, under the four population ethics criteria under study.

${ }^{31}$ This is the case because, when $\gamma=1$, the denominator of the first factor of the RHS is 0 , and the second factor is negative, so that the RHS equals $-\infty$. Hence $\alpha$ cannot be smaller than the RHS, and thus the condition on $\alpha$ is never satisfied.

${ }^{32}$ To see here why a dilution of lifetime on more people may be socially desirable under NDU, we can, here again, compute the welfare change from a rise in $L$ compensated by a fall in $S$ in such a way as to maintain the total lifetime constant, i.e. $\frac{\partial W^{c}}{\partial L}=\frac{\partial W}{\partial L}+\frac{\partial W}{\partial S} \frac{\partial S}{\partial L}$ :

$$
L^{\gamma-1}\left(\frac{Q}{L}\right)^{\sigma}\left[\frac{\gamma-\sigma+(1-\gamma) S^{1-\sigma}-(1-\sigma) S^{-\sigma}}{\left(1-S^{1-\sigma}\right)^{2}}\right]-\frac{L^{\gamma-1} \alpha(1-\gamma)}{1-S}
$$

The term in brackets is negative. Hence, given that the second term is also negative if $\alpha>0$, we have that $\frac{\partial W^{c}}{\partial L}<0$ under $\alpha \geq 0$. But once $\alpha<0$, the second term is positive, and adding new persons while reducing $S$ may be welfare-increasing, as under AU. 


\begin{tabular}{|c|c|c|c|c|}
\hline \multicolumn{5}{|c|}{ Table 1: The spaceship problem without reproduction } \\
\hline & Parameters & $Q$ & $\alpha$ & $\sigma$ \\
\hline Criteria & $\begin{array}{c}\text { Impact on } \\
\{L, S, 0\} \succeq\{\tilde{L}, \tilde{S}, 0\}\end{array}$ & & & \\
\hline $\mathrm{CU}$ & & no & no & no \\
\hline $\mathrm{AU}$ & & no if $\alpha \geq 0$ & no if $\alpha \geq 0$ & no if $\alpha \geq 0$ \\
\hline & & yes if $\alpha<0$ & yes if $\alpha<0$ & yes if $\alpha<0$ \\
\hline CLU & & no & no & no \\
\hline NDU & & no if $\alpha \geq 0$ & no if $\alpha \geq 0$ & no if $\alpha \geq 0$ \\
\hline & & yes if $\alpha<0$ & yes if $\alpha<0$ & yes if $\alpha<0$ \\
\hline
\end{tabular}

Table 1 illustrates the major division between our four normative criteria: whereas rankings of lifetime-equal histories under CU and CLU are invariant to the total available space, as well as to individual preferences, the same is not true under AU and NDU once $\alpha$ is negative. Therefore, the sign of the intercept of the temporal utility function is a major discriminant factor among our normative criteria. When $\alpha \geq 0$, the social planner selects the lifetime-equal history with the lowest initial population and the largest survival rate, under all utilitarian criteria. However, if $\alpha<0$, the solutions under AU and NDU become dependent on the total available space and the preference parameter $\sigma$.

In sum, there are two distinct ways to interpret Table 1. On the one hand, one may start from what one regards as a morally relevant or a simply empirically observable piece of information, and select, on the basis of that, a normative criterion. On the other hand, one may, from the start, opt for one criterion, and then consider what affects the solution under that particular view.

If one has no obvious reason to believe in a particular population ethics criterion a priori, Table 1 will be interpreted in the former way, and one can then advocate in favour of CU or CLU on several distinct grounds. For instance, $\mathrm{CU}$ and CLU do not require any information on individual preferences to give a solution to the spaceship problem without reproduction. Given the difficulty to estimate $\alpha$ empirically, the invariance of rankings to $\alpha$ can be regarded as a strong argument in favour of CU and CLU. Alternatively, one may argue that the solution to the spaceship problem should be invariant to the spaceship size $Q$, and defend CU and CLU on those grounds. ${ }^{33}$

\section{The spaceship problem (2): reproduction}

Let us now turn to the more general problem where the population of the spaceship can reproduce itself. This amounts to relaxing the postulate of a zero fertility rate $n$ (i.e. Assumption A11). That more general spaceship problem can be regarded as including the widely debated issue of the optimal population existing on the Earth. After all, the Earth can be regarded as a spaceship with living conditions allowing the reproduction of its population.

To study the spaceship problem with reproduction, we will, here again, examine how a social planner ranks lifetime-equal histories $\{L, S, n\}$ and $\{\tilde{L}, \tilde{S}, \tilde{n}\}$.

\footnotetext{
${ }^{33}$ The intuition supporting the property of invariance to $Q$ is the following: once the total time to be lived is fixed, why should the discovery of a new place to live (e.g. a new galaxy) question the past selection of the best history? Given the - so far unfinished - exploration of the Universe, one may want the current solution of the spaceship problem - i.e. given the currently known $Q$ - to remain valid even if one discovers a new place in the future.
} 
For the ease of presentation, we consider here the comparison of lifetime-equal histories with the same initial population sizes (i.e. $L=\tilde{L}$ ), that is, the number of pioneers is equal in all histories. Such a way to proceed is actually quite natural, as the population of the Earth (or any other spaceship) 'is what it is', so that a social planner governing a spaceship has no other choice than to take the initial population $L$ as given. Indeed, from a policy perspective, the only possible interventions concern future fertility $n$ or longevity $1 / 1-S$, but one can hardly change the initial population size $L$. Thus assuming common initial populations $L=\tilde{L}$ seems plausible.

- Assumption A12 The initial population size $L$ is equal in all histories.

Under $L=\tilde{L}$, and assuming a finite number of life-periods (i.e. $S n<1$ ), lifetime-equal histories $\{L, S, n\}$ and $\{\tilde{L}, \tilde{S}, \tilde{n}\}$ are such that

$$
\frac{1}{(1-S)(1-n S)}=\frac{1}{(1-\tilde{S})(1-\tilde{n} \tilde{S})}
$$

A history with longer lives will also exhibit, for a given total number of life-periods, a lower fertility: $S \geq \tilde{S} \Longleftrightarrow n \leq \tilde{n}$. In the spaceship problem with reproduction, comparing two lifetime-equal histories $\{L, S, n\}$ and $\{L, \tilde{S}, \tilde{n}\}$ amounts to comparing two histories for which:

$$
\frac{1-\tilde{S}}{1-S}=\frac{1-n S}{1-\tilde{n} \tilde{S}}
$$

that is, there is an equality of the ratios of strength of mortality [i.e. $1-S$ ] (LHS) and strengths of fertility [i.e. $\frac{1}{1-n S}$ ] (RHS) for histories $\{L, S, n\}$ and $\{L, \tilde{S}, \tilde{n}\}$ having an equal number of life-periods (i.e. for which $P=\tilde{P}$ ). Intuitively, if one history exhibits a larger fertility (i.e. $\frac{1}{1-\tilde{n} \tilde{S}}>\frac{1}{1-n S}$ ) than another history with the same total number of life-periods, it must also be characterized by a larger strength of mortality (i.e. $1-\tilde{S}>1-S$ ).

\subsection{The Classical Utilitarian solution}

As in the problem without reproduction, it is tempting to believe, at first glance, that $\mathrm{CU}$ will exhibit a populationist bias, and lead to the selection of a large fertility rate and a low survival rate. However, Proposition 5 suggests that this is not necessarily the case.

Proposition 5 Assume A1-A10 and A12. Consider two lifetime-equal histories $\{L, S, n\}$ and $\{L, \tilde{S}, \tilde{n}\}$, with $S<\tilde{S}$ and $n>\tilde{n}$. The $C U$ planner prefers $\{L, S, n\}$ to $\{L, \tilde{S}, \tilde{n}\}$ if and only if:

$$
\sum_{t=0}^{\infty}\left(S^{t} \frac{1-n^{t+1}}{1-n}\right)^{1-\sigma} \geq \sum_{t=0}^{\infty}\left(\tilde{S}^{t} \frac{1-\tilde{n}^{t+1}}{1-\tilde{n}}\right)^{1-\sigma}
$$

Proof. See the Appendix.

As in the problem without reproduction, the rankings under CU are independent from the size of the spaceship $Q$. Moreover, the social ordering of lifetimeequal histories is also independent from the intercept $\alpha$. Furthermore, the initial 
size of the population, $L$, has no effect on the social ordering over lifetime-equal histories. Thus whatever the initial population is, CU ranks lifetime-equal histories in the same manner. For those who appreciate the independence of rankings from the past, that property of $\mathrm{CU}$ is highly valuable.

However, note that, despite some common aspects with the spaceship problem without reproduction (e.g. independence of the solution from $Q$ and $\alpha$ ), there exists, nonetheless, a fundamental difference between Propositions 1 and 5. Whereas CU leads, in the absence of reproduction, to the selection of the history with the lowest $L$ and the highest $S$, it is difficult here to see whether CU recommends a population with a high survival and a low fertility or the opposite. Different combinations of $n$ and $S$ yield different intertemporal paths of $q_{t}$, and the ranking of lifetime-equal histories depends on how sensitive temporal welfare is to the space available for each person (i.e. the parameter $\sigma$ ).

Thus, the introduction of reproduction on the spaceship alters the conclusions drawn in the no reproduction case. Under $0<\sigma<1$, it is now hard to see whether a CU planner would opt for a pre-demographic transition state (i.e. with a high $n$ and a low $S$ ), or, alternatively, for a post-demographic transition state (i.e. with a low $n$ and a high $S$ ), as long as the total number of life-periods is the same, whereas, in the problem without reproduction, the CU solution always consisted in choosing the highest survival rate.

Finally, note that it is nonetheless possible to see what the CU social ranking in the extreme cases where $\sigma=0$ and $\sigma=1 .^{34}$

- Corollary C3 Under $\sigma=0$, the CU planner is indifferent between any lifetime-equal histories.

- Corollary C4 Under $\sigma=1$, the CU planner is indifferent between any lifetime-equal histories.

\subsection{Average Utilitarianism}

AU yields an ambiguous ranking in the absence of reproduction, depending on the intercept of the temporal utility function $\alpha$. Once reproduction is allowed, things do not become more clear, as stated in Proposition 6.

Proposition 6 Assume A1 to A10 and A12. Consider two lifetime-equal histories $\{L, S, n\}$ and $\{L, \tilde{S}, \tilde{n}\}$, with $S<\tilde{S}$ and $n>\tilde{n}$. The AU planner prefers $\{L, S, n\}$ to $\{L, \tilde{S}, \tilde{n}\}$ if and only if:

$\sum_{t=0}^{\infty}\left(S^{t} \frac{1-n^{t+1}}{1-n}\right)^{1-\sigma}-\frac{1-S}{1-\tilde{S}} \sum_{t=0}^{\infty}\left(\tilde{S}^{t} \frac{1-\tilde{n}^{t+1}}{1-\tilde{n}}\right)^{1-\sigma} \geq \frac{\alpha Q^{-\sigma} L^{\sigma}}{1-S n}\left[\frac{1}{1-\tilde{S}}-\frac{1}{1-S}\right]$

or, in terms of $\alpha$,

$\alpha \leq \frac{Q^{\sigma} L^{-\sigma}(1-S n)(1-\tilde{S})(1-S)}{\tilde{S}-S}\left[\sum_{t=0}^{\infty}\left(S^{t} \frac{1-n^{t+1}}{1-n}\right)^{1-\sigma}-\frac{1-S}{1-\tilde{S}} \sum_{t=0}^{\infty}\left(\tilde{S}^{t} \frac{1-\tilde{n}^{t+1}}{1-\tilde{n}}\right)^{1-\sigma}\right]$

Proof. See the Appendix.

Contrary to Proposition 5, three factors affect the social ranking of lifetimeequal histories. First, the intercept of the temporal utility function $\alpha$; second,

\footnotetext{
${ }^{34}$ See the Appendix for the proof.
} 
the total space available $Q$; third, the initial population size $L$. All this makes the AU solution likely to differ from the CU solution. Let us briefly examine the impact of those three determinants of the social ordering.

The impact of $\alpha$ depends on its sign. Clearly, whatever $\alpha$ is positive or negative, the LHS of the first condition in Proposition 6 is smaller than the LHS in Proposition 5. Hence, if $\alpha \geq 0$, AU recommends a lifetime-equal history with a higher $S$ and a lower $n$ in comparison with the one under CU. However, under $\alpha<0$, the RHS of the first condition in Proposition 6 becomes negative, so that it is no longer certain that AU selects a lifetime-equal history with a higher $S$ and a lower $n$ than CU. We may actually observe the opposite, because of the dilution effect mentioned in the problem without reproduction.

Regarding the impact of the available space $Q$, it is easy to see that the smaller $Q$ is, the larger the RHS of the first condition in Proposition 6 is, so that lifetime-equal histories with a high $S$ and a low $n$ are more likely to be selected (under $\alpha \geq 0$ ). The opposite prevails under $\alpha$ sufficiently negative, on the grounds of the dilution effect (see above).

As far as the influence of the initial population $L$ is concerned, Proposition 6 suggests that the larger $L$ is, the larger the RHS of the first condition for $\{L, S, n\} \succeq\{L, \tilde{S}, \tilde{n}\}$ is, so that $\mathrm{AU}$ is more likely to select a lifetime-equal history with a high $S$ and a low $n$, under $\alpha \geq 0$. However, under $\alpha<0$, a larger $L$ may make the dilution of misery necessary, leading to a lifetime-equal history with a high $n$ and a low $S$. The AU solution is thus, unlike the CU solution, dependent on the initial population size, and, thus, dependent on the past.

Finally, it should be noticed that the AU solution differs here from what it is in the absence of reproduction. True, as without reproduction, AU does not necessarily lead to the selection of the lifetime-equal history with the lowest population size and the largest life expectancy when temporal welfare is negative. But, more importantly, even for a non-negative $\alpha$ is the selection of lifetime-equal histories with the highest $S$ no longer certain here, contrary to what prevailed in the absence of reproduction.

\subsection{Critical-Level Utilitarianism}

The preferences of a CLU social planner over lifetime-equal histories $\{L, S, n\}$ and $\{L, \tilde{S}, \tilde{n}\}$ are characterized by Proposition 7 .

Proposition 7 Assume A1 to A10 and A12. Consider two lifetime-equal histories $\{L, S, n\}$ and $\{L, \tilde{S}, \tilde{n}\}$, with $S<\tilde{S}$ and $n>\tilde{n}$. The $C L U$ planner prefers $\{L, S, n\}$ to $\{L, \tilde{S}, \tilde{n}\}$ if and only if:

$\sum_{t=0}^{\infty}\left(S^{t} \frac{1-n^{t+1}}{1-n}\right)^{1-\sigma}-\sum_{t=0}^{\infty}\left(\tilde{S}^{t} \frac{1-\tilde{n}^{t+1}}{1-\tilde{n}}\right)^{1-\sigma} \geq\left(\frac{Q}{L}\right)^{-\sigma} \hat{u}\left(\frac{1}{1-n S}-\frac{1}{1-\tilde{n} \tilde{S}}\right)$

Proof. See the Appendix.

Note that, under $\hat{u}=0$, the condition stated in Proposition 7 collapses to the condition under $\mathrm{CU}$ (Proposition 5). Note also that, under $\hat{u}$ equal to average lifetime welfare, the condition for $\{L, S, n\} \succeq\{L, \tilde{S}, \tilde{n}\}$ coincides with the condition under AU (Proposition 6). Therefore, we can interpret CLU as a natural generalization of $\mathrm{CU}$ and $\mathrm{AU}$.

Having stressed this, it remains that the CLU solution to the spaceship problem remains somewhat closer, in some sense, of the $\mathrm{CU}$ one in comparison to 
the AU one. The reason why this is so is that the condition stated in Proposition 7 does not depend on $\alpha$, exactly like the condition under CU. That result is somewhat surprising, as one would expect the ranking under CLU to depend on how large the critical level $\hat{u}$ is with respect to the intercept $\alpha$. But here again, and exactly as in the case of $\mathrm{CU}$, the level of the intercept of the temporal utility function does not matter.

However, the proximity of the CLU and CU solutions has some limits. Firstly, the size of the initial population $L$ influences the solution of the spaceship problem here, unlike under CU. The larger $L$ is, the more likely the preference for a lifetime-equal history with a lower fertility and a lower mortality is. Thus, the extent to which the demographic transition is valuable depends, under a fixed available space, on the size of the population that undergoes the transition. The larger the population is, the more beneficial the demographic transition is. Secondly, note that the solution to the spaceship problem with reproduction depends, under CLU, on the spaceship size $Q$, unlike what it was the case under $\mathrm{CU}$. This constitutes another important distinction between the two normative criteria in the present context.

\subsection{Number-Dampened Utilitarianism}

Let us now consider the rankings induced by NDU over lifetime-equal histories.

Proposition 8 Assume $A 1$ to $A 10$ and A12. Consider two lifetime-equal histories $\{L, S, n\}$ and $\{L, \tilde{S}, \tilde{n}\}$, with $\tilde{S}>S$ and $n>\tilde{n}$. The NDU planner prefers $\{L, S, n\}$ to $\{L, \tilde{S}, \tilde{n}\}$ if and only if:

$$
\begin{aligned}
& \sum_{t=0}^{\infty}\left(S^{t} \frac{1-n^{t+1}}{1-n}\right)^{1-\sigma}-\left(\frac{1-S}{1-\tilde{S}}\right)^{1-\gamma} \sum_{t=0}^{\infty}\left(\tilde{S}^{t} \frac{1-\tilde{n}^{t+1}}{1-\tilde{n}}\right)^{1-\sigma} \\
\geq & \frac{\alpha(Q)^{-\sigma} L^{\sigma}}{(1-S n)^{1-\gamma}}\left[\left(\frac{1}{1-\tilde{S} \tilde{n}}\right)^{\gamma} \frac{1}{1-\tilde{S}}-\left(\frac{1}{1-S n}\right)^{\gamma} \frac{1}{1-S}\right]
\end{aligned}
$$

or, in terms of $\alpha$,

$\alpha \leq \frac{Q^{\sigma} L^{-\sigma}(1-S n)^{1-\gamma}\left[\sum_{t=0}^{\infty}\left(S^{t} \frac{1-n^{t+1}}{1-n}\right)^{1-\sigma}-\left(\frac{1-S}{1-\tilde{S}}\right)^{1-\gamma} \sum_{t=0}^{\infty}\left(\tilde{S}^{t} \frac{1-\tilde{n}^{t+1}}{1-\tilde{n}}\right)^{1-\sigma}\right]}{\left(\frac{1}{1-\tilde{S} \tilde{n}}\right)^{\gamma} \frac{1}{1-\tilde{S}}-\left(\frac{1}{1-S n}\right)^{\gamma} \frac{1}{1-S}}$

Proof. See the Appendix.

If $\gamma$ equals 1 , the condition for $\{L, S, n\} \succeq\{L, \tilde{S}, \tilde{n}\}$ coincides with the one under CU (Proposition 5), while, under $\gamma$ equal to 0 , we are back to the AU condition (Proposition 6). Thus, exactly as in the absence of reproduction, NDU is, like CLU, a generalization of standard utilitarian criteria.

However, a significant difference remains between the two criteria: the ordering under CLU over lifetime-equal histories is invariant to the intercept of the temporal utility function $\alpha$, contrary to the ordering under NDU, which depends on whether $\alpha$ is positive or negative, large or small. That result does not surprise us, as this was already present in the simpler version of the spaceship problem considered in Section 3. But the present analysis reveals that this fundamental difference between CLU and NDU is robust to the introduction of reproduction on the spaceship. 


\subsection{A synthesis}

To sum up, Table 2 shows the determinants of the social ranking of lifetime-equal histories in the spaceship problem with reproduction.

\begin{tabular}{cccccc}
\hline Table 2: & The spaceship problem & with reproduction \\
& Parameters & $Q$ & $L$ & $\alpha$ & $\sigma$ \\
Criteria & Impact on & & & & \\
& $\{L, S, n\} \succeq\{L, \tilde{S}, \tilde{n}\}$ & & & & \\
$\mathrm{CU}$ & & no & no & no & yes \\
$\mathrm{AU}$ & yes & yes & yes & yes \\
$\mathrm{CLU}$ & yes & yes & no & yes \\
NDU & yes & yes & yes & yes \\
\hline \hline
\end{tabular}

Table 2 illustrates that our four normative criteria are not sensitive to the same information. The CU solution is the only one that is invariant to the spaceship size $Q$ and to the initial population $L$. Hence, for decision-makers who would like their decisions to be independent from the past, CU seems to be the adequate criterion. The three other criteria depend all on how large the initial population is, and on how large the spaceship is. But those three remaining criteria differ from each others, as the AU and the NDU solutions depend on the intercept of the temporal utility function $\alpha$, contrary to the CLU solution. Therefore if one considers that $\alpha$ cannot be observed, and, thus, cannot serve as a basis for the social planner's ranking, it follows that, if $Q$ and $L$ are regarded as relevant, only the CLU solution is adequate. Finally, note that the AU and NDU solutions are both dependent on the four factors explicitly listed in Table 2. Thus those two normative criteria are equally demanding in terms of information, and differ only in how that information is treated.

The comparison of Table 2 (spaceship with reproduction) with Table 1 (spaceship without reproduction) shows that the parameter $\sigma$, which captures the sensitivity of temporal welfare to space per head, plays now a crucial role whatever the criterion is, unlike what was the case in the absence of reproduction, where $\sigma$ was benign (except under a negative $\alpha$ under AU and NDU).

The role of other parameters is also strengthened once reproduction is introduced. The total space available $Q$ was, in general, benign in the absence of reproduction (except under a negative $\alpha$ under AU and NDU), but once reproduction is allowed, $Q$ becomes a major variable for all normative criteria except CU. Moreover, the intercept $\alpha$ plays now a significant role than in the spaceship problem without reproduction.

\section{The spaceship problem (3): stationary space- ship}

While we focused so far on a spaceship whose population would only enjoy, as a whole, a finite total number of life-periods, that restriction may appear quite pessimistic, as this implies a population that will vanish asymptotically towards 0 (even though it may exhibit some growth during a part of history). That finiteness assumption, although analytically convenient, presupposed the impossibility for Mankind to have an infinite history, because of some constraints 
preventing the perpetual survival of life in the spaceship. In the case of humanmade spaceships like rockets, this constraint would follow, for instance, from a limit in the quantity of oxygen available. But in the case of more complex spaceships with ecosystems (like the Earth), this restriction seems less plausible.

If one believes in human capacity to overcome technical difficulties, the finiteness assumption $S n<1$ must be relaxed. This is the reason why we will here depart from A10, and consider instead the case of a stationary spaceship, that is, a spaceship with a finite constant asymptotic population. Actually, it is not difficult to see that the constancy of the asymptotic population requires, in this framework, nothing else than $n S=1$. Indeed, the constancy of the population at a given point in time requires an equality of births and deaths

$$
\begin{aligned}
L\left(S^{t} n^{t}\right) & =\frac{L\left(S^{t} n^{t}\right)}{S n}(1-S)+\frac{L\left(S^{t} n^{t}\right)}{S^{2} n^{2}} S(1-S)+\ldots+L S^{t-1}(1-S) \\
& =\frac{L\left(S^{t} n^{t}\right)}{S n}(1-S)\left[1+\frac{1}{n}+\frac{1}{n^{2}}+\ldots+\frac{1}{n^{t-1}}\right]
\end{aligned}
$$

Hence a constant asymptotic population (i.e. $t \rightarrow \infty$ ) requires

$$
1=\frac{1-S}{S n-S}
$$

If $\frac{1-S}{S n-S}>1$, the population tends to zero asymptotically, as the long-run number of deaths exceeds the long-run number of births. On the contrary, if $\frac{1-S}{S n-S}<1$, the population tends to infinity, as the long-run number of births exceeds the long-run number of deaths. Finally, it is only under $\frac{1-S}{S n-S}=1$, that is, under $S n=1$, that the population is asymptotically constant. This is the assumption we will make in this section.

- Assumption A13 The population of the spaceship converges asymptotically towards a positive constant, i.e. $S n=1$.

Note that, under that assumption, the long-run level of the population of the spaceship will be exactly equal to the total number of life-periods under no reproduction on the spaceship. That equivalence result is stated below.

Proposition 9 Under an initial population $L$, a survival rate $S$ and a fertility rate $n$ such that $n S=1$, the long-run population size equals the total number of life-periods in the absence of reproduction, i.e. $\frac{L}{1-S}$.

Proof. See the Appendix.

The intuition behind that equivalence result is the following. Both numbers - the total number of life-periods under $n=0$ and the total population size - have, at time 0 , the same level, equal to $L$. The number of periods under $n=0$ becomes, at time 1 , equal to $L+L S$, while the number of persons under $n S=1$ becomes, at time 1 , equal to $S L+S L n$, which is $S L+L$. At $t=2$, the total number of periods in the spaceship without reproduction is $L+S L+S^{2} L$, while the number of persons under $n S=1$ is $S^{2} L+S^{2} L n+S^{2} L n^{2}$, that is, $S^{2} L+S L+L$, and so forth. We are thus in presence of two sums whose terms are the same, yielding Proposition 9.

Having stressed that equivalence result, there remains a fundamental difference between a spaceship without reproduction and a stationary spaceship: 
while the former involves a finite number of life-periods, the latter involves an infinite number of life-periods. Formally, the total number of life-periods is

$$
P=\frac{L}{(1-S)(1-S n)}
$$

so that, under $S n=1$, the number of life-periods is infinite. Hence any two histories $\{L, S, n\}$ and $\{\tilde{L}, \tilde{S}, \tilde{n}\}$ with $S n=\tilde{S} \tilde{n}=1$ have an infinite $P$.

The non-finiteness of total lifetimes is really problematic for an aggregative doctrine like utilitarianism, whatever the precise form it takes, because such an aggregative doctrine aims at taking into account the utilities at all lifeperiods, so that the infinity of life-periods makes the utilitarian planner deal with quantities that are no longer finite. There exist two broad families of solutions to that problem. On the one hand, one can depart from utilitarianism, and turn towards a less aggregative ethical standard (e.g. adopting a Maximin objective focusing on the worse-off cohort). On the other hand, one may keep the utilitarian doctrine, but restrict the informational basis that is taken into account.

In the rest of this section, we will take the second road, and remain within utilitarianism. What we shall do is to reduce social welfare comparisons to what prevails at the stationary state, that is, at the population level that, once reached, will prevail forever in the spaceship. In other words, we will concentrate on the living conditions faced by all generations succeeding to each others once the steady-state is reached, each generation enjoying a finite life, and giving birth to another generation that will also enjoy exactly the same living conditions (i.e. same space per head). That focus on the stationary state is a way to 'extract' the spaceship from time, that is, from history. This means that all things occurring outside the steady-state will not be taken into account here. ${ }^{35}$

Therefore, in order to characterize the solutions of the stationary spaceship problem, we will, in this section, focus on histories that are not only lifetimeequal, which is the case of all histories under $S n=1$, but on histories that are population-equal, in the sense that these yield the same long-run population.

- Definition D7 Two histories $\{L, S, n\}$ and $\{\tilde{L}, \tilde{S}, \tilde{n}\}$ are population-equal if these yield the same asymptotic population size, that is, if and only if

$$
\frac{L}{1-S}=\frac{\tilde{L}}{1-\tilde{S}}
$$

Note that, given $S n=1$, the comparison of population-equal histories amounts to focusing on two demographic parameters: the size of the pioneer group, $L$, and the survival proportion $S$. Here again, this leads us back, in some way, to the spaceship problem without reproduction: comparing two population-equal histories $\{L, S, n\}$ and $\{\tilde{L}, \tilde{S}, \tilde{n}\}$ amounts to comparing histories $\{L, S, 1 / S\}$ and $\{\tilde{L}, \tilde{S}, 1 / \tilde{S}\}$ with $\frac{L}{1-S}=\frac{\tilde{L}}{1-\tilde{S}}$, exactly as the spaceship problem without reproduction amounts to comparing histories $\{L, S, 0\}$ and $\{\tilde{L}, \tilde{S}, 0\}$ with $\frac{L}{1-S}=\frac{\tilde{L}}{1-\tilde{S}}$.

\footnotetext{
${ }^{35}$ Naturally, such a reduction, which is allowed by the existence of a state of constant population, is far from neutral on ethical grounds: ignoring a whole span of history and concentrating on a 'timeless' spaceship is obviously a simplification. However, given that the stationary state will be faced by an infinite number of people, the reduction of history to the timeless stationary spaceship seems legitimate, as a first approximation.
} 


\subsection{The Instantaneous View}

Let us now examine how two population-equal histories $\{L, S, 1 / S\}$ and $\{\tilde{L}, \tilde{S}, 1 / \tilde{S}\}$, with $L>\tilde{L}$ and $S<\tilde{S}$, are ranked by a utilitarian social planner focusing on the stationary state of the spaceship.

For that purpose, we will first consider a planner who compares populationequal histories by investigating which one yields the largest instantaneous social welfare at the steady-state, that is, the largest social welfare during one period of time, once the spaceship is stationary in population size.

- Definition D8 A social planner adheres to the Instantaneous View (IV), if, when comparing two population-equal histories $\{L, S, 1 / S\}$ and $\{\tilde{L}, \tilde{S}, 1 / \tilde{S}\}$ with $L>\tilde{L}$ and $S<\tilde{S}$, he prefers the history yielding the highest social welfare during one period of time at the steady-state, i.e. $W^{*}$ :

$$
\{L, S, 1 / S\} \succeq\{\tilde{L}, \tilde{S}, 1 / \tilde{S}\} \Longleftrightarrow W^{*}(L, S, 1 / S) \geq W^{*}(\tilde{L}, \tilde{S}, 1 / \tilde{S})
$$

As stated in Proposition 10, IV yields to a general indifference between all population-equal histories, whatever the utilitarian criterion used. ${ }^{36}$

Proposition 10 Assume A1 to A9 and A13. Consider two population-equal histories $\{L, S, 1 / S\}$ and $\{\tilde{L}, \tilde{S}, 1 / \tilde{S}\}$, with $L>\tilde{L}$ and $S<\tilde{S}$. Under $C U, A U$, $C L U$ or $N D U$, an $I V$ planner regards $\{L, S, 1 / S\}$ and $\{\tilde{L}, \tilde{S}, 1 / \tilde{S}\}$ as equivalent.

Proof. See the Appendix.

Proposition 10 is a negative result, as it is of the 'anything goes' type. Certainly all population-equal histories should not be regarded as equivalent. Having a population of long-lived or short-lived people, cannot be considered as the same thing, even though the total number of people alive in the long-run is the same. Thus reducing the informational basis to the mere steady-state social welfare per period is a far too strong reduction, which makes all population-equal histories look the same, despite their strong demographic differences.

Those differences in fertility and longevity are ignored here, as the histories compared are characterized by an equal long-run population size, which is the only piece of demographic information that matters from an IV perspective. Thus, one should not expect from IV to be able to discriminate between population-equal histories.

In order to avoid this 'anything goes' result, an alternative solution is to define social welfare as the sum of the lifetime welfares of the members of the long-run population, instead of their instantaneous welfares. This approach will be coined the 'posterity view'.

\subsection{The Posterity View}

The intuition behind that extension of the informational basis is merely that, even though the asymptotic population size is equal in two histories $\{L, S, 1 / S\}$ and $\{\tilde{L}, \tilde{S}, 1 / \tilde{S}\}$, whether a life is, on average, longer in the latter than in the former is not something irrelevant, but, rather, should be taken into account.

\footnotetext{
${ }^{36}$ Indeed, each utilitarian criterion is only sensitive to the population size (which is equal in all histories), and not to its age-structure.
} 
- Definition D9 A social planner adheres to the Posterity View (PV), if, when comparing two population-equal histories $\{L, S, 1 / S\}$ and $\{\tilde{L}, \tilde{S}, 1 / \tilde{S}\}$ with $L>\tilde{L}$ and $S<\tilde{S}$, he prefers the history yielding the highest lifetime welfare for a cohort, the spaceship being in its stationary state

$$
\{L, S, 1 / S\} \succeq\{\tilde{L}, \tilde{S}, 1 / \tilde{S}\} \Longleftrightarrow \frac{W^{*}(L, S, 1 / S)}{1-S} \geq \frac{W^{*}(\tilde{L}, \tilde{S}, 1 / \tilde{S})}{1-\tilde{S}}
$$

As stated below, the PV approach yields a preference for population-equal histories with a small initial population and a large survival rate when $\alpha \geq 0$. Under $\alpha<0$, histories with a smaller initial population size and a larger life expectancy are better only if temporal utility at the stationary state is nonnegative. Otherwise, PV selects histories with a large $L$ and a low $S$, to dilute the pain of existence on the largest number of persons.

Proposition 11 Assume A1 to A9 and A13. Consider two population-equal histories $\{L, S, 1 / S\}$ and $\{\tilde{L}, \tilde{S}, 1 / \tilde{S}\}$, with $L>\tilde{L}$ and $S<\tilde{S}$. Under $C U, A U$, $C L U$ or $N D U$, the preferences of a $P V$ planner are:

- If $\alpha \geq 0,\{\tilde{L}, \tilde{S}, 1 / \tilde{S}\} \succeq\{L, S, 1 / S\}$, whatever $Q$ and $\sigma$ are.

- If $\alpha<0,\{\tilde{L}, \tilde{S}, 1 / \tilde{S}\} \succeq\{L, S, 1 / S\}$ if and only if $-\alpha \leq\left(\frac{Q(1-S)}{L}\right)^{\sigma}$.

Proof. See the Appendix.

Take any two population-equal histories. In the first one, there is a large number of pioneers and a small proportion of survivors and, thus, given $n S=$ 1 , a large fertility rate. In that history, people live short lives, and are, on average, young. In the second one, there is a small number of pioneers, a large proportion of survivors, and a low fertility rate, so that, in that second history, people are, on average, older. At the steady-state, the two populations have the same sizes, but different age-structures. If we only look at the aggregated instantaneous welfare at the steady-state, the age-structure does not matter: anything goes. But if one regards the aggregated lifetime welfare at the steadystate, the ranking varies with the level of $\alpha$.

When $\alpha \geq 0$, the history with the longest lives must be better. The reason why this is the case is merely that the first history can only achieve a large population by a large 'turnover' rate. But a high turnover rate is associated with shorter lives, which is here damageable on a lifetime perspective, given that temporal welfare is non-negative. Hence population-equal histories with such a high turnover cannot meet posterity utilitarian requirements.

On the contrary, when $\alpha<0$, a history with the lowest turnover is not necessarily better. If temporal utility is negative, it is better to have the highest turnover, and, hence, to opt for the first history. Note that whether temporal utility is positive or negative at the stationary state depends on the postulated asymptotic population size $\frac{L}{1-S}$. The higher this is, the more likely is the preference for a history with a large turnover and a low life expectancy, in such a way as to dilute pain on the largest number of persons.

\subsection{The Renewal View}

After having studied the IV, yielding an overall indifference between populationequal histories, and the PV, which yields more ambiguous results, but, for $\alpha$ 
sufficiently large, a preference for histories with long lives, it may be worthwhile, for completeness, to consider the third alternative: the one in which, for a given temporal welfare, the social planner prefers to observe a perpetual renewal of the spaceship. Of course, under a stationary population, such a renewal of the population can only come from the replacement of old members of the spaceship. One can thus hardly advocate for renewal while condemning the turnover. The posterity view and the renewal view are incompatible.

In the spaceship, the number of deaths at the stationary state is a fraction $1-S$ of the whole population, and is thus equal to $(1-S) \frac{L}{1-S}=L$. There are $L$ persons leaving the spaceship at each period, and, given the stationary population size, these are replaced by $L$ new persons, who are actually born from the survivors of the previous births, whose size is now $L S$, so that the newborns are equal to $L S n=L$. Given that $L$ is both the number of arrivals and the number of departures from the stationary spaceship, it is a natural measure of renewal. This can be used to weight temporal utility, the intuition being that the lower the renewal rate is, the lower the weight assigned to temporal utility.

- Definition D10 A social planner adheres to the Renewal View (RV), if, when comparing two population-equal histories $\{L, S, 1 / S\}$ and $\{\tilde{L}, \tilde{S}, 1 / \tilde{S}\}$ with $L>\tilde{L}$ and $S<\tilde{S}$, he prefers the history yielding the highest renewalweighted welfare for a cohort, the spaceship being in its stationary state

$$
\{L, S, 1 / S\} \succeq\{\tilde{L}, \tilde{S}, 1 / \tilde{S}\} \Longleftrightarrow L W^{*}(L, S, 1 / S) \geq \tilde{L} W^{*}(\tilde{L}, \tilde{S}, 1 / \tilde{S})
$$

Proposition 12 presents the ranking of population-equal histories under RV.

Proposition 12 Assume A1 to A9 and A13. Consider two population-equal histories $\{L, S, 1 / S\}$ and $\{\tilde{L}, \tilde{S}, 1 / \tilde{S}\}$, with $L>\tilde{L}$ and $S<\tilde{S}$. Under $C U, A U$, $C L U$ or $N D U$, the preferences of an $R V$ planner are:

- If $\alpha \geq 0,\{L, S, 1 / S\} \succeq\{\tilde{L}, \tilde{S}, 1 / \tilde{S}\}$, whatever $Q$ and $\sigma$ are.

- If $\alpha<0,\{L, S, 1 / S\} \succeq\{\tilde{L}, \tilde{S}, 1 / \tilde{S}\}$ if and only if $-\alpha \leq\left(\frac{Q(1-S)}{L}\right)^{\sigma}$.

Proof. See the Appendix.

Adopting RV leads to a social ordering that is contrary to the one under $\mathrm{PV}$. When $\alpha \geq 0$, the social planner prefers population-equal histories where the renewal of the group is the largest, and where life expectancy is the shortest. However, when $\alpha$ is very negative, making temporal utility negative, it is better to avoid a large turnover, as it makes misery spread on a larger set of beings. Hence, in that case, histories with longer lives are better, as these minimize the turnover, and, thus, misery (as measured under RV).

Note that, under $\alpha<0$, the larger the available space $Q$ is, the more likely is the preference for a history with a high turnover. The preference parameter $\sigma$ plays the same role. In sum, the above condition amounts to requiring a nonnegative temporal utility, as otherwise, it is socially optimal to raise longevity as much as possible, in such a way as to minimize the turnover, and, thus, the spread of misery on potential beings.

Whether RV recommends a large or a low turnover depends on the asymptotic population size $\frac{L}{1-S}$ : the higher it is, the more likely is the preference for a history with a low turnover, and a high life expectancy, which is exactly the opposite of the recommendation under PV. 
Finally, let us notice that, if the social planner wanted both to adopt a lifecycle view and to weight the calculated individual lifetime welfare by the renewal measure proposed here, the outcome would coincide with what IV recommends, that is, an indifference between all population-equal histories. One can thus interpret IV as an exact compromise between PV and RV.

\subsection{A synthesis}

To summarize this section, Table 3 shows the impact of $Q, \alpha$ and $\sigma$ on the social ordering over population-equal histories, under IV, PV and RV. Normative population criteria such as CU, AU, CLU and NDU do not help any more here, as the arbitrages between population-equal histories lie outside differentnumbers comparison. The crucial issue at stake concerns how individual welfare is counted: either one can take instantaneous utility (i.e. IV), or weight instantaneous utility by a lifetime index (i.e. PV), or by a turnover index (i.e. RV).

\begin{tabular}{|c|c|c|c|c|}
\hline \multicolumn{5}{|c|}{ Table 3: The stationary spaceship problem } \\
\hline \multirow{3}{*}{ Criteria } & Parameters & $Q$ & $\alpha$ & $\sigma$ \\
\hline & Impact on & & & \\
\hline & $\{L, S, 1 / S\} \succeq\{\tilde{L}, \tilde{S}, 1 / \tilde{S}\}$ & & & \\
\hline IV & & no & no & no \\
\hline $\mathrm{PV}$ & & no if $\alpha \geq 0$ & no if $\alpha \geq 0$ & no if $\alpha \geq 0$ \\
\hline & & yes if $\alpha<0$ & yes if $\alpha<0$ & yes if $\alpha<0$ \\
\hline $\mathrm{RV}$ & & no if $\alpha \geq 0$ & no if $\alpha \geq 0$ & no if $\alpha \geq 0$ \\
\hline & & yes if $\alpha<0$ & yes if $\alpha<0$ & yes if $\alpha<0$ \\
\hline
\end{tabular}

A first observation to be made is that IV is the unique approach under which the social ordering over population-equal histories is independent from $Q, \alpha$ and $\sigma$. This invariance may be regarded as a strength of that approach, but it has also its drawbacks: whatever those parameters are, all populationequal histories are regarded as equivalent under IV. We are thus left with no precise answer to our question, as all histories are regarded as equally good.

To avoid this 'anything goes' result, it is tempting to consider the two alternatives: the PV and the RV approaches. Each of these yields an ordering over population-equal histories that takes a simple form under $\alpha \geq 0$ : either a preference for histories with a high lifetime and a low turnover (under PV), or a preference for histories with a short lifetime and a high turnover (under $\mathrm{RV})$. In each case, the social ordering does not depend on $Q$, or on $\sigma$, so that those solutions have a high level of generality. Moreover, these solutions are also independent from the asymptotic population size. However, when $\alpha<0$, the social ordering over population-equal histories under PV and RV may depend on $Q, \alpha$ and $\sigma$. Furthermore, it is straightfoward to see from Propositions 11 and 12 that, under PV and RV, the social ordering of population-equal histories depends on the level of the asymptotic population size.

In sum, the focus on a spaceship with a constant asymptotic population size does not make the selection of a history easier. Either one adheres to the IV approach, but then one cannot discriminate between any population-equal histories, or one adheres to the PV or RV approach, which allow a ranking of 
population-equal histories, but such a ranking has also its cost: it requires a larger informational basis, including estimates of $\sigma$ (and possibly $\alpha$ ). Hence relaxing the fixed resources constraint, and, thus, shifting from a finite to a potentially infinite history, does not simplify the problem.

\section{Concluding remarks}

The goal of this paper was to cast new light on the spaceship problem, by paying particular attention to the trade-offs between adding new persons and extending the life of existing persons. For that purpose, we assumed that individual lifetime welfare is additive over time, and that temporal welfare depends negatively on population density, and we characterized, under those assumptions, the preference orderings of a utilitarian social planner over lifetime-equal histories, which all exhibit, by construction, an equal number of life-periods.

In the spaceship problem without reproduction, CU selects, contrary to its populationist reputation, lifetime-equal histories with the smallest number of persons and the largest longevity. CLU yields exactly the same ordering, independently from the critical utility level, which is another counterintuitive result. $\mathrm{AU}$ and NDU take, unlike the two former criteria, more information into account under a negative intercept of the temporal utility function, and, quite surprisingly, may exhibit a bias for larger populations in comparison with CU.

Once reproduction is introduced on the spaceship, the social ordering over lifetime-equal histories is no longer as clear as without reproduction. Here our normative criteria yield quite different rankings, as these do not rely on the same informational basis. CU is the only criterion of our list that ranks histories independently from the initial population size and the total spaceship size. Moreover, whereas $\mathrm{CU}$ and CLU regard the intercept of the temporal utility function as irrelevant, the same is not true for AU and NDU.

Then, our focus on the stationary spaceship problem - where all histories being now characterized by an infinite number of life-periods - forced us to shift our attention from the comparison of lifetime-equal histories to the comparison of population-equal histories. Given that standard utilitarian criteria do, in that context, generate the same rankings of population-equal histories, additional pieces of information must be incorporated into the value function of the planner, to account for intuitions related to the value of posterity and renewal. We showed that the instantaneous view (IV) can be regarded as a compromise between those two opposite intuitions: posterity (PV) and renewal (RV).

In sum, whereas the population ethics literature examined, in general, the ranking of populations differing in size and in welfare per person (see Blackorby et al 2005), this paper shows that changing the fundamentals on which population ethics relies, by introducing fertility and longevity, and by making welfare dependent on space, does not leave population debates unaffected. Firstly, populationist or antipopulationist biases usually associated to criteria such as $\mathrm{CU}$ and $\mathrm{AU}$ can be questioned in the light of our study. Secondly, and more importantly, our analysis highlighted the differentiated influences of economic fundamentals (individual preferences, total available space, existing population, etc.) on the social ranking of histories under distinct utilitarian criteria. Given that the existing literature relied on other fundamentals, the (in)variance of population orderings to those fundamentals could hardly be examined before. 
By complementing the existing literature on population ethics, this paper brings also a contribution to the current debates on the future population of the Earth. Clearly, the population has always been at the centre of intergenerational justice debates, and of the design of a sustainable mode of development (see Sikora and Barry 1978; Broome 1992). One can indeed hardly talk about long-run development without evoking demographic trends, and the pressure the demography can put on the welfare of individuals. Our analysis, in which demographic variables are explicit, can thus serve as a basis for discussing the selection of an adequate normative criterion aimed at solving population dilemmas. As such, this constitutes the first, necessary stage before deriving population policies aimed at improving social welfare.

Finally, while our re-examination of the spaceship problem casts new light on that old issue, it should be stressed that further refinements of this analysis could be made, to explore the robustness of our conclusions. Assumptions such as temporal utility strictly increasing in space per head for any space level, and the additivity of lifetime welfare, may be questioned. Hence this work is only a first stage in the re-examination of the spaceship problem, which invites others.

\section{Acknowledgments}

The authors are most grateful to Gustaf Arrhenius, Pierre Pestieau and two anonymous referees for helpful suggestions and comments on this paper.

\section{References}

Arrhenius, G. \& Bykvist, K. (1995). Interpersonal compensation and moral duties to future generations: moral aspects of energy use. Uppsala: Uppsala Prints and Preprints in Philosophy, 21, Department of Philosophy, Uppsala University.

Arrhenius, G. (2008). Life extension versus replacement. Journal of Applied Philosophy, $25(3), 211-227$.

Bentham, J. (1789). Introduction to the Principles of Morals and Legislation. In M. Warnock (Ed.), Utilitarianism (1962). London: Fontana Press.

Blackorby, C. \& Donaldson, D. (1984). Social criteria for evaluating population change. Journal of Public Economics, 25, 13-33.

Blackorby, C., Bossert, W., \& Donaldson, D. (2005). Population Issues in Social Choice Theory, Welfare Economics, and Ethics. Cambridge: Cambridge University Press.

Bommier, A. (2006). Uncertain lifetime and intertemporal choice: risk aversion as a rationale for time discounting. International Economic Review, 47(4), 1223-1246.

Botero, G. (1588). Della Cause della Grandezza della Citta. Reprinted partly in The Population and Development Review, 11(2), 1985, 335-340.

Boulding, K. (1966). The Earth as a spaceship. Washington State University Commitee on Space Sciences, available at

http://www.colorado.edu/econ/Kennneth.Boulding/spaceship-earth.html.

Broome, J. (1992). Counting the Cost of Global Warming. Cambridge: The White Horse Press.

Broome, J. (2004). Weighing Lives. Oxford: Oxford University Press.

Broome, J. (2007). Replies. Economics and Philosophy, 23, 115-124. 
Bykvist, K. (2007). The good, the bad and the ethically neutral. Economics and Philosophy, 23, 97-105.

Cantillon, R. (1755). Essay on the Nature of Trade in General. Paris: INED (new edition: 1952).

Cramer, V., Torgersen, S., \& Kringlen, E. (2004). Quality of life in a city: the effect of population density. Social Indicators Research, 69, 103-116.

Crisp, R. (2007). Neutrality and pleasure. Economics and Philosophy, 23, 81-88.

Gottlieb, M. (1945). The theory of optimum population for a closed economy. Journal of Political Economy, 53, 289-316.

Heiling, G.K. (1994). How many people can be fed on Earth? In W. Lutz (Ed.), The Future Population of the World. What can we assume today? London: IIASA, Earthscan.

Hurka, T. (1983). Value and population size. Ethics, 93, April, 496-507.

Kremer, M. (1993). Population growth and technological change: one million BC to 1990. Quarterly Journal of Economics, 108, 681-716.

Malthus, T.R. (1798). Essay on the Principle of Population. London: Pelican Books (reprint: 1970).

Malthus, T.R. (1830). A Summary View. London: Pelican Books (reprint: 1970).

Mill, J.S. (1859). On Liberty. Edited by M. Warnock (1962). London: Fontana Press.

Ng, Y.-K. (1986). Social criteria for evaluating population change: an alternative to the Blackorby-Donaldson criterion. Journal of Public Economics, 29, 375-381.

Parfit, D. (1984). Reasons and Persons. Oxford: Oxford University Press.

Ramsey, F.P. (1928). A mathematical theory of saving. Economic Journal, 38, 543-549.

Schumpeter, J.A. (1954). A History of Economic Analysis. London: George Allen \& Unwin Ltd.

Sidgwick, H. (1874). The Methods of Ethics. London: MacMillan.

Sikora, R.I. \& Barry, B. (1978). Obligations to Future Generations. Temple University Press.

Weitzman, M. (1998). The Noah's Ark Problem. Econometrica, 66(6), 1279-1298.

\section{Appendix}

\subsection{The spaceship problem (1)}

Proposition 1 - The Classical Utilitarian solution Social welfare is larger under $\{L, S, 0\}$ than under $\{\tilde{L}, \tilde{S}, 0\}$ if and only if:

$$
L\left(\frac{Q}{L}\right)^{\sigma} \frac{1}{1-S^{1-\sigma}}+L \frac{\alpha}{1-S} \geq \tilde{L}\left(\frac{Q}{\tilde{L}}\right)^{\sigma} \frac{1}{1-\tilde{S}^{1-\sigma}}+\tilde{L} \frac{\alpha}{1-\tilde{S}}
$$

Given that $\frac{L}{1-S}=\frac{\tilde{L}}{1-\tilde{S}}$, the second terms of the LHS and RHS cancel each other. Moreover, as $\frac{L}{1-S}=\frac{\tilde{L}}{1-\tilde{S}}$, we have $\left(\frac{L}{\tilde{L}}\right)^{1-\sigma}=\left(\frac{1-S}{1-\tilde{S}}\right)^{1-\sigma}$. Thus social welfare is larger under $\{L, S, 0\}$ than under $\{\tilde{L}, \tilde{S}, 0\}$ if and only if

$$
\frac{(1-S)^{1-\sigma}}{1-S^{1-\sigma}} \geq \frac{(1-\tilde{S})^{1-\sigma}}{1-\tilde{S}^{1-\sigma}}
$$

The following Lemma will be useful in showing that the above inequality is never satisfied for $\tilde{S}>S$. 
Lemma 13 Consider the function $F(S)=\frac{(1-S)^{a}}{1-S^{b}}$ with $0<b<1$ and $a \leq b$. We have $F^{\prime}(S) \geq 0$ for all $0<S<1$.

Proof. Applying the quotient rule of differentiation, it follows that:

$$
\begin{aligned}
F^{\prime}(S) & =\frac{a(1-S)^{a-1}(-1)\left(1-S^{b}\right)-(1-S)^{a}\left(-b S^{b-1}\right)}{\left(1-S^{b}\right)^{2}} \\
& =\frac{(1-S)^{a-1} a}{\left(1-S^{b}\right)^{2}}\left[\frac{b}{a} S^{b-1}(1-S)-\left(1-S^{b}\right)\right]
\end{aligned}
$$

Note that $S^{b-1}>0,(1-S)>0$, and $\left(1-S^{b}\right)>0$.

First consider $a<0$, in which case we have not only $\frac{(1-S)^{a-1} a}{\left(1-S^{b}\right)^{2}}<0$ but also $\left[\frac{b}{a} S^{b-1}(1-S)-\left(1-S^{b}\right)\right]<0$ as well, implying $F^{\prime}(S)>0$.

If $a=0$, we have $\frac{(1-S)^{a-1} a}{\left(1-S^{b}\right)^{2}}=0$, immediately implying $F^{\prime}(S)=0$.

Finally, consider $a>0$. In this case, $\frac{(1-S)^{a-1} a}{\left(1-S^{b}\right)^{2}}>0$, so that the sign of $F^{\prime}(S)$ is the same as the sign of $\left[\frac{b}{a} S^{b-1}(1-S)-\left(1-S^{b}\right)\right]$. Since $S^{b-1}(1-$ $S)>0$, this expression is clearly decreasing in $a$. Thus, if it can be shown that $\left[\frac{b}{a} S^{b-1}(1-S)-\left(1-S^{b}\right)\right]$ is positive at the largest acceptable value of $a$ (i.e. $a=b)$, then it would follow that $\left[\frac{b}{a} S^{b-1}(1-S)-\left(1-S^{b}\right)\right]$ is positive for all acceptable values of $a$. With $a=b$, this expression becomes $\left[S^{b-1}(1-S)-\left(1-S^{b}\right)\right]=S^{b-1}-S^{b}-1+S^{b}=S^{b-1}-1$. Since $0<S<1$ and $0<b<1$ (so that $b-1<0$ ), it follows that $S^{b-1}>1$. Therefore $S^{b-1}-1>0$, implying $F^{\prime}(S)>0$ for all $0<a \leq b$.

That Lemma can be used to characterize the social planner's preferences over two lifetime-equal histories $\{L, S, 0\}$ and $\{\tilde{L}, \tilde{S}, 0\}$. Indeed, we know that $\{L, S, 0\}$ is better than $\{\tilde{L}, \tilde{S}, 0\}$ if and only if:

$$
\frac{(1-S)^{1-\sigma}}{1-S^{1-\sigma}} \geq \frac{(1-\tilde{S})^{1-\sigma}}{1-\tilde{S}^{1-\sigma}}
$$

From the Lemma, it follows that $\frac{(1-S)^{1-\sigma}}{1-S^{1-\sigma}}$ is increasing in $S$ (since $\frac{(1-S)^{1-\sigma}}{1-S^{1-\sigma}}$ is the specification of $F(S)$ with $a=b=1-\sigma)$. Therefore $\{L, S, 0\} \preceq\{\tilde{L}, \tilde{S}, 0\}$.

Regarding Corollary 1 , note that, under $\sigma=0$, the inequality $\frac{(1-S)^{1-\sigma}}{1-S^{1-\sigma}} \geq$ $\frac{(1-\tilde{S})^{1-\sigma}}{1-\tilde{S}^{1-\sigma}}$ is always a strict equality, so that indifference prevails between all lifetime-equal histories.

Regarding Corollary 2, note that, under $\sigma=1$, social welfare is

$$
\begin{aligned}
& L\left(\frac{Q}{L}+\alpha\right)+S L\left(\frac{Q}{S L}+\alpha\right)+S^{2} L\left(\frac{Q}{S^{2} L}+\alpha\right)+\ldots \\
= & Q+Q+\ldots+Q+\frac{\alpha L}{1-S}
\end{aligned}
$$

That expression is the same for all lifetime-equal histories. 
Proposition 2 - The Average Utilitarian solution Under AU, social welfare under a history $\{L, S, 0\}$ is

$$
\begin{aligned}
W & =\frac{L\left(\frac{Q}{L}\right)^{\sigma}+L \alpha+S L\left(\frac{Q}{S L}\right)^{\sigma}+S L \alpha+S^{2} L\left(\frac{Q}{S^{2} L}\right)^{\sigma}+S^{2} L \alpha+\ldots}{L} \\
W & =\left(\frac{Q}{L}\right)^{\sigma} \frac{1}{1-S^{1-\sigma}}+\frac{\alpha}{1-S}
\end{aligned}
$$

Take the case where $\alpha \geq 0$. It is straightforward to see that

$$
\begin{aligned}
\frac{\partial W}{\partial S} & =\left(\frac{Q}{L}\right)^{\sigma} \frac{1-\sigma}{\left(1-S^{1-\sigma}\right)^{2} S^{\sigma}}+\frac{\alpha}{(1-S)^{2}}>0 \\
\frac{\partial W}{\partial L} & =Q^{\sigma}(-\sigma) L^{-\sigma-1} \frac{1}{1-S^{1-\sigma}}<0
\end{aligned}
$$

Thus, any increase in $S$ is preferred by an AU planner, and any decrease in $L$ is preferred by an AU planner. Therefore, such a planner would prefer $\{\tilde{L}, \tilde{S}, 0\}$ to $\{L, S, 0\}$ with $L>\tilde{L}$ and $S<\tilde{S}$ even without any restriction on the relation between $L$ and $S$. Hence an AU planner prefers histories with a higher survival rate and a smaller initial population in a sense much stronger than a CU planner, since for an AU planner such a preference arises even if the two different profiles of demographic parameters do not yield lifetime-equal histories.

In the case where $\alpha<0$, the same general conclusions cannot be drawn. Actually, under $\alpha<0$, it is still true that $\frac{\partial W}{\partial L}<0$, but not necessarily true that $\frac{\partial W}{\partial S}>0$. Hence one cannot conclude that an AU planner necessarily prefers a history with a higher survival rate, unlike under $\alpha \geq 0$.

If one focuses on lifetime-equal histories $\{L, S, 0\}$ and $\{\tilde{L}, \tilde{S}, 0\}$ with $L>\tilde{L}$ and $S<\tilde{S}$, it is possible to derive a general condition on $\alpha$ such that $\{L, S, 0\}$ is preferred to $\{\tilde{L}, \tilde{S}, 0\}$.

Indeed, social welfare is larger under $\{L, S, 0\}$ than under $\{\tilde{L}, \tilde{S}, 0\}$ if and only if:

$$
\left(\frac{Q}{L}\right)^{\sigma} \frac{1}{1-S^{1-\sigma}}+\frac{\alpha}{1-S} \geq\left(\frac{Q}{\tilde{L}}\right)^{\sigma} \frac{1}{1-\tilde{S}^{1-\sigma}}+\frac{\alpha}{1-\tilde{S}}
$$

Given that $\frac{L}{1-S}=\frac{\tilde{L}}{1-\tilde{S}}$, we have:

$$
\frac{Q^{\sigma} L^{-\sigma}}{(1-S)^{-\sigma}}\left[\frac{(1-S)^{-\sigma}}{1-S^{1-\sigma}}-\frac{(1-\tilde{S})^{-\sigma}}{1-\tilde{S}^{1-\sigma}}\right] \geq \alpha\left[\frac{1}{1-\tilde{S}}-\frac{1}{1-S}\right]
$$

By the Lemma, the factor in brackets is always strictly negative, so that the LHS is negative. ${ }^{37}$ We know that the second factor of the RHS is positive, and so, given $\alpha<0$, the sign of the RHS is also negative. Thus the above inequality is true if and only if $\alpha$ is sufficiently negative, that is, if and only if

$$
\alpha \leq \frac{Q^{\sigma} L^{-\sigma}(1-\tilde{S})(1-S)}{(\tilde{S}-S)(1-S)^{-\sigma}}\left[\frac{(1-S)^{-\sigma}}{1-S^{1-\sigma}}-\frac{(1-\tilde{S})^{-\sigma}}{1-\tilde{S}^{1-\sigma}}\right]
$$

as stated in Proposition 2.

\footnotetext{
${ }^{37}$ Note that, under $\alpha \geq 0$, that inequality is thus never satisfied, as the LHS is strictly negative, and the RHS is strictly positive. Hence, in conformity with the above findings, $\{L, S, 0\} \prec\{\tilde{L}, \tilde{S}, 0\}$.
} 
Proposition 3 - The Critical-Level Utilitarian solution Social welfare is larger under $\{L, S, 0\}$ than under $\{\tilde{L}, \tilde{S}, 0\}$ if and only if:

$$
L\left(\frac{Q}{L}\right)^{\sigma} \frac{1}{1-S^{1-\sigma}}+L \frac{\alpha}{1-S}-L \hat{u} \geq \tilde{L}\left(\frac{Q}{\tilde{L}}\right)^{\sigma} \frac{1}{1-\tilde{S}^{1-\sigma}}+\tilde{L} \frac{\alpha}{1-\tilde{S}}-\tilde{L} \hat{u}
$$

Given that $\frac{\tilde{L}}{1-\tilde{S}}=\frac{L}{1-S}$, that condition becomes:

$$
\frac{(1-S)^{1-\sigma}}{1-S^{1-\sigma}} \geq \frac{(1-\tilde{S})^{1-\sigma}}{1-\tilde{S}^{1-\sigma}}+\frac{\hat{u}(L-\tilde{L})(1-\tilde{S})^{1-\sigma}}{\tilde{L}^{1-\sigma} Q^{\sigma}}
$$

Given the Lemma, the first term of the RHS exceeds the LHS under $\tilde{S}>S$. Hence, given that the second term of the RHS is strictly positive, the above inequality is never satisfied, and $\{\tilde{L}, \tilde{S}, 0\}$ is always preferred to $\{L, S, 0\}$.

Proposition 4 - The Number-Dampened Utilitarian solution Social welfare is larger under $\{L, S, 0\}$ than under $\{\tilde{L}, \tilde{S}, 0\}$ if and only if:

$$
(Q)^{\sigma}\left[\frac{L^{\gamma-\sigma}}{1-S^{1-\sigma}}-\frac{\tilde{L}^{\gamma-\sigma}}{1-\tilde{S}^{1-\sigma}}\right] \geq \alpha\left[\frac{\tilde{L}^{\gamma}}{1-\tilde{S}}-\frac{L^{\gamma}}{1-S}\right]
$$

Given that $\frac{\tilde{L}}{1-\tilde{S}}=\frac{L}{1-S}$, that condition becomes:

$$
\frac{(Q)^{\sigma} L^{\gamma-\sigma}}{(1-S)^{\gamma-\sigma}}\left[\frac{(1-S)^{\gamma-\sigma}}{1-S^{1-\sigma}}-\frac{(1-\tilde{S})^{\gamma-\sigma}}{1-\tilde{S}^{1-\sigma}}\right] \geq \frac{\alpha L^{\gamma}}{(1-S)^{\gamma}}\left[\frac{(1-\tilde{S})^{\gamma}}{1-\tilde{S}}-\frac{(1-S)^{\gamma}}{1-S}\right]
$$

By the Lemma, we know that $\frac{(1-S)^{\gamma-\sigma}}{1-S^{1-\sigma}}$ is increasing in $S$ (since $\frac{(1-S)^{\gamma-\sigma}}{1-S^{1-\sigma}}$ is the specification of $F(S)$ with $a=\gamma-\sigma$ and $b=1-\sigma$ ). Hence, given $\tilde{S}>S$, the LHS is necessarily negative. Regarding the RHS, we know also, still by using our Lemma, that $\frac{(1-S)^{\gamma}}{1-S}$ is increasing in $S$ (since $\frac{(1-S)^{\gamma}}{1-S}$ is the specification of $F(S)$ with $a=\gamma$ and $b=1$ ), so that the factor in brackets in the RHS is positive.

Hence, two cases should be distinguished, depending on $\alpha$.

If $\alpha \geq 0$, the LHS is negative and the RHS is positive, so that the above inequality is never satisfied. Thus, under $\alpha \geq 0$, we have that $\{\tilde{L}, \tilde{S}, 0\}$ is always preferred to $\{L, S, 0\}$.

If $\alpha<0$, the LHS is negative and the RHS is also negative, so that the above condition may be satisfied. Actually, isolating $\alpha$ from the above inequality yields:

$$
\alpha \leq \frac{Q^{\sigma} L^{-\sigma}(1-\tilde{S})(1-S)}{(1-S)^{\gamma-\sigma}\left[(1-\tilde{S})^{\gamma}(1-S)^{1-\gamma}-(1-\tilde{S})\right]}\left[\frac{(1-S)^{\gamma-\sigma}}{1-S^{1-\sigma}}-\frac{(1-\tilde{S})^{\gamma-\sigma}}{1-\tilde{S}^{1-\sigma}}\right]
$$

which is the condition appearing in Proposition 4.

Note that, if $\gamma=0$, that condition is the same as under AU, whereas, under $\gamma=1$, that condition is never satisfied, as the denominator of the first factor of the RHS equals 0 , so that the RHS equals $-\infty$. Thus $\alpha$ cannot be smaller than the RHS, and so the condition is never satisfied, and $\{\tilde{L}, \tilde{S}, 0\}$ is always preferred to $\{L, S, 0\}$ as under CU. 


\subsection{The spaceship problem (2)}

Proposition 5 - The Classical Utilitarian solution When counting the welfare of each cohort separately, social welfare is

$$
\begin{aligned}
& L\left[\left(\frac{Q}{L}\right)^{\sigma}+S\left(\frac{Q}{S L+n S L}\right)^{\sigma}+S^{2}\left(\frac{Q}{S^{2} L+n S^{2} L+n^{2} S^{2} L}\right)^{\sigma}+\ldots\right]+L \alpha(1+S+\ldots) \\
& +S L n\left[\left(\frac{Q}{S L+n S L}\right)^{\sigma}+S\left(\frac{Q}{S^{2} L+n S^{2} L+n^{2} S^{2} L}\right)^{\sigma}+\ldots\right]+S L n \alpha(1+S+\ldots) \\
& +S^{2} L n^{2}\left[\left(\frac{Q}{S^{2} L+n S^{2} L+n^{2} S^{2} L}\right)^{\sigma}+\ldots\right]+S^{2} L n^{2} \alpha(1+S+\ldots)+\ldots
\end{aligned}
$$

If one sorts out the two components of temporal welfare, one has:

$$
\begin{aligned}
& L\left[\left(\frac{Q}{L}\right)^{\sigma}+S\left(\frac{Q}{L}\right)^{\sigma}(S+S n)^{-\sigma}+S^{2}\left(\frac{Q}{L}\right)^{\sigma}\left(S^{2}+n S^{2}+n^{2} S^{2}\right)^{-\sigma}+\ldots\right] \\
& +S L n\left[\left(\frac{Q}{L}\right)^{\sigma}(S+S n)^{-\sigma}+S\left(\frac{Q}{L}\right)^{\sigma}\left(S^{2}+n S^{2}+n^{2} S^{2}\right)^{-\sigma}+\ldots\right] \\
& +\ldots+\frac{L \alpha}{1-S}+\frac{S L n \alpha}{1-S}+\frac{S^{2} L n^{2} \alpha}{1-S}+\ldots
\end{aligned}
$$

Therefore, social welfare is

$$
\begin{aligned}
& L\left(\frac{Q}{L}\right)^{\sigma}\left[1+S^{1-\sigma}(1+n)^{-\sigma}+S^{2(1-\sigma)}\left(1+n+n^{2}\right)^{-\sigma}+\ldots\right] \\
& +\operatorname{Ln}\left(\frac{Q}{L}\right)^{\sigma}\left[S^{1-\sigma}(1+n)^{-\sigma}+S^{2(1-\sigma)}\left(1+n+n^{2}\right)^{-\sigma}+S^{3(1-\sigma)}\left(1+n+n^{2}+n^{3}\right)^{-\sigma}+\ldots\right] \\
& +\operatorname{Ln}^{2}\left(\frac{Q}{L}\right)^{\sigma}\left[S^{2(1-\sigma)}\left(1+n+n^{2}\right)^{-\sigma}+\ldots\right]+\ldots+\frac{L \alpha}{(1-S)(1-S n)}
\end{aligned}
$$

Hence, noting that $\sum_{s=0}^{t} n^{s}=\frac{1-n^{t+1}}{1-n}$, we have

$$
L\left(\frac{Q}{L}\right)^{\sigma}+L\left(\frac{Q}{L}\right)^{\sigma} S^{1-\sigma}\left(\frac{1-n^{2}}{1-n}\right)^{1-\sigma}+\ldots+\frac{L \alpha}{(1-S)(1-S n)}
$$

Note that, by definition of lifetime-equal histories, we have: $\frac{L}{(1-S)(1-S n)}=$ $\frac{L}{(1-\tilde{S})(1-\tilde{S} \tilde{n})}$ for any two lifetime-equal histories $\{L, S, n\}$ and $\{L, \tilde{S}, \tilde{n}\}$. Hence the last term does not affect the social ranking of those histories.

Therefore social welfare is larger under $\{L, S, n\}$ than under $\{L, \tilde{S}, \tilde{n}\}$ if and only if:

$$
\sum_{t=0}^{\infty}\left(\frac{S^{t}-S^{t} n^{t+1}}{1-n}\right)^{1-\sigma} \geq \sum_{t=0}^{\infty}\left(\frac{\tilde{S}^{t}-\tilde{S}^{t} \tilde{n}^{t+1}}{1-\tilde{n}}\right)^{1-\sigma}
$$

The corollary follows from fixing $\sigma=0$ in the above condition. This yields:

$$
\sum_{t=0}^{\infty} S^{t} \frac{1-n^{t+1}}{1-n} \geq \sum_{t=0}^{\infty} \tilde{S}^{t} \frac{1-\tilde{n}^{t+1}}{1-\tilde{n}}
$$

which is a strict equality, as the LHS and the RHS are equal to the total number of life-periods per initial resident of the spaceship, in, respectively, $\{L, S, n\}$ and 
$\{L, \tilde{S}, \tilde{n}\}$, which is, by definition, the same in all lifetime-equal histories. Thus it follows that $\{L, S, n\} \sim\{L, \tilde{S}, \tilde{n}\}$.

Moreover, social welfare is, under $\sigma=1$ :

$$
\begin{aligned}
& \frac{L Q}{L}+\frac{(S L+n S L) Q}{S L+n S L}+\frac{\left(S^{2} L+n S^{2} L+n^{2} S^{2} L\right) Q}{S^{2} L+n S^{2} L+n^{2} S^{2} L}+\ldots+\frac{L \alpha}{(1-S)(1-S n)} \\
= & Q+Q+\ldots+Q+\frac{L \alpha}{(1-S)(1-S n)}
\end{aligned}
$$

Hence social welfare is here the same in all lifetime-equal histories.

Proposition 6 - The Average Utilitarian solution Under AU, social welfare is

$$
\begin{aligned}
& \frac{L\left(\frac{Q}{L}\right)^{\sigma}+(S L+n S L)\left(\frac{Q}{S L+n S L}\right)^{\sigma}+\ldots}{L+L S n+L S^{2} n^{2}+\ldots}+\frac{\frac{\alpha L}{(1-S)(1-n S)}}{L+L S n+L S^{2} n^{2}+\ldots} \\
= & (1-S n)\left(\frac{Q}{L}\right)^{\sigma}\left[\sum_{t=0}^{\infty}\left(S^{t} \sum_{s=0}^{t} n^{s}\right)^{1-\sigma}\right]+\frac{\alpha}{1-S}
\end{aligned}
$$

Given $\sum_{s=0}^{t} n^{s}=\frac{1-n^{t+1}}{1-n}$, social welfare under $\{L, S, n\}$ is larger than under $\{L, \tilde{S}, \tilde{n}\}$ if and only if:

$$
\sum_{t=0}^{\infty}\left(S^{t} \frac{1-n^{t+1}}{1-n}\right)^{1-\sigma}-\frac{1-S}{1-\tilde{S}} \sum_{t=0}^{\infty}\left(\tilde{S}^{t} \frac{1-\tilde{n}^{t+1}}{1-\tilde{n}}\right)^{1-\sigma} \geq \frac{\alpha Q^{-\sigma} L^{\sigma}}{1-S n}\left[\frac{1}{1-\tilde{S}}-\frac{1}{1-S}\right]
$$

Isolating $\alpha$, that condition becomes:

$$
\alpha \leq \frac{Q^{\sigma} L^{-\sigma}(1-S n)(1-\tilde{S})(1-S)}{\tilde{S}-S}\left[\sum_{t=0}^{\infty}\left(S^{t} \frac{1-n^{t+1}}{1-n}\right)^{1-\sigma}-\frac{1-S}{1-\tilde{S}} \sum_{t=0}^{\infty}\left(\tilde{S}^{t} \frac{1-\tilde{n}^{t+1}}{1-\tilde{n}}\right)^{1-\sigma}\right]
$$

Proposition 7 - The Critical-Level Utilitarian solution Social welfare is here equal to

$$
\begin{aligned}
& L\left(\frac{Q}{L}\right)^{\sigma}+(S L+n S L)\left(\frac{Q}{S L+n S L}\right)^{\sigma}+\left(S^{2} L+n S^{2} L+n^{2} S^{2} L\right)\left(\frac{Q}{S^{2} L+n S^{2} L+n^{2} S^{2} L}\right)^{\sigma}+\ldots \\
& +\frac{L}{(1-S)(1-S n)} \alpha-\left(L+S L n+S^{2} L n^{2}+\ldots\right) \hat{u} \\
= & L\left(\frac{Q}{L}\right)^{\sigma}\left[1+(S(1+n))^{1-\sigma}+\left(S^{2}\left(1+n+n^{2}\right)\right)^{1-\sigma}+\ldots\right]+L \frac{\alpha}{(1-S)(1-S n)}-L \frac{1}{1-n S} \hat{u}
\end{aligned}
$$

Hence, social welfare is larger under $\{L, S, n\}$ than under $\{L, \tilde{S}, \tilde{n}\}$ if and only if:

$$
\sum_{t=0}^{\infty}\left(S^{t} \frac{1-n^{t+1}}{1-n}\right)^{1-\sigma}-\sum_{t=0}^{\infty}\left(\tilde{S}^{t} \frac{1-\tilde{n}^{t+1}}{1-\tilde{n}}\right)^{1-\sigma} \geq\left(\frac{Q}{L}\right)^{-\sigma} \hat{u}\left(\frac{1}{1-n S}-\frac{1}{1-\tilde{n} \tilde{S}}\right)
$$


Proposition 8 - The Number-Dampened Utilitarian solution Social welfare is here equal to

$$
\begin{aligned}
& {\left[L+S L n+S^{2} L n^{2}+\ldots\right]^{\gamma}\left[(1-S n)\left(\frac{Q}{L}\right)^{\sigma}\left[\sum_{t=0}^{\infty}\left(S^{t} \sum_{s=0}^{t} n^{s}\right)^{1-\sigma}\right]+\frac{\alpha}{1-S}\right] } \\
= & L^{\gamma-\sigma}(1-S n)^{1-\gamma}(Q)^{\sigma}\left[\sum_{t=0}^{\infty}\left(S^{t} \sum_{s=0}^{t} n^{s}\right)^{1-\sigma}\right]+\left[\frac{L}{1-S n}\right]^{\gamma} \frac{\alpha}{1-S}
\end{aligned}
$$

Hence, social welfare is larger under $\{L, S, n\}$ than under $\{L, \tilde{S}, \tilde{n}\}$ if and only if:

$$
\begin{aligned}
& \sum_{t=0}^{\infty}\left(S^{t} \frac{1-n^{t+1}}{1-n}\right)^{1-\sigma}-\left(\frac{1-S}{1-\tilde{S}}\right)^{1-\gamma}\left[\sum_{t=0}^{\infty}\left(\tilde{S}^{t} \frac{1-\tilde{n}^{t+1}}{1-\tilde{n}}\right)^{1-\sigma}\right] \\
\geq & \frac{\alpha(Q)^{-\sigma} L^{\sigma}}{(1-S n)^{1-\gamma}}\left[\left(\frac{1}{1-\tilde{S} \tilde{n}}\right)^{\gamma} \frac{1}{1-\tilde{S}}-\left(\frac{1}{1-S n}\right)^{\gamma} \frac{1}{1-S}\right]
\end{aligned}
$$

\subsection{The spaceship problem (3)}

Proposition 9 - The equivalence result If $n=0$, the total number of life-periods is:

$$
L+S L+S^{2} L+\ldots=\frac{L}{1-S}
$$

Knowing that the born at time $t$ are equal to the born at time $t-1$ multiplied by $S n$, the long-run population is (from the youngest to the oldest)

$L_{\infty}=\left(L S^{\infty} n^{\infty}\right) S n+\left(L S^{\infty-1} n^{\infty-1}\right) S n+\ldots+\left(L S^{2} n^{2}\right) S^{\infty-2}+(L S n) S^{\infty-1}+L S^{\infty}$

Under $S n=1$, this vanishes to

$$
L+L S+L S^{2}+L S^{3}+\ldots+L S^{\infty}=\frac{L}{1-S}
$$

Proposition 10 - The Instantaneous View Two population-equal histories have, by definition, the same stationary asymptotic population, equal to $\frac{L}{1-S}$. Thus these have the same space per head $\left(q=\frac{Q(1-S)}{L}\right)$, and the same utility per life-period. Therefore, those histories have the same social welfare. For instance, social welfare at the steady-state is, under $\mathrm{CU}$, equal in the two histories, as:

$$
\frac{L}{1-S}\left[\left(\frac{Q(1-S)}{L}\right)^{\sigma}+\alpha\right]=\frac{\tilde{L}}{1-\tilde{S}}\left[\left(\frac{Q(1-\tilde{S})}{\tilde{L}}\right)^{\sigma}+\alpha\right]
$$

as long as $\frac{L}{1-S}=\frac{\tilde{L}}{1-\tilde{S}}$. This result holds also under other utilitarian criteria, as these yield the same rankings as $\mathrm{CU}$ as long as the population size is the same, independently from whether that population is made of young or old persons. 
Proposition 11 - The Posterity View The average lifetime welfare at the steady-state is the product of life expectancy and utility per life-period: $\frac{1}{1-S}\left[\left(\frac{Q(1-S)}{L}\right)^{\sigma}+\alpha\right]$. Thus steady-state social welfare is, under CU, larger in $\{\tilde{L}, \tilde{S}, 1 / \tilde{S}\}$ than in $\{L, S, 1 / S\}$ if and only if:

$$
\frac{\tilde{L}}{1-\tilde{S}} \frac{1}{1-\tilde{S}}\left[\left(\frac{Q(1-\tilde{S})}{\tilde{L}}\right)^{\sigma}+\alpha\right] \geq \frac{L}{1-S} \frac{1}{1-S}\left[\left(\frac{Q(1-S)}{L}\right)^{\sigma}+\alpha\right]
$$

Given that $\frac{L}{1-S}=\frac{\tilde{L}}{1-\tilde{S}}$ and $\frac{1}{1-\tilde{S}}>\frac{1}{1-S}$, that condition is $\left(\frac{Q(1-S)}{L}\right)^{\sigma} \geq-\alpha$. That condition is a strict equality when $\alpha \geq 0$, so that we have $\{\tilde{L}, \tilde{S}, 1 / \tilde{S}\}$ better than $\{L, S, 1 / S\}$. But if $\alpha<0$, that is no longer true. We have $\{L, S, 1 / S\}$ better than $\{\tilde{L}, \tilde{S}, 1 / \tilde{S}\}$ if and only if $\left(\frac{Q(1-S)}{L}\right)^{\sigma}<-\alpha$. A similar rationale can be used for AU, CLU and NDU.

Proposition 12 - The Renewal View The temporal welfare of a person living at the steady-state, if weighted by the renewal index $L$, is $L\left[\left(\frac{Q(1-S)}{L}\right)^{\sigma}+\alpha\right]$. Hence social welfare at the steady-state is, under CU, larger in $\{L, S, 1 / S\}$ than in $\{\tilde{L}, \tilde{S}, 1 / \tilde{S}\}$ if and only if:

$$
\frac{L}{1-S} L\left[\left(\frac{Q(1-S)}{L}\right)^{\sigma}+\alpha\right] \geq \frac{\tilde{L}}{1-\tilde{S}} \tilde{L}\left[\left(\frac{Q(1-\tilde{S})}{\tilde{L}}\right)^{\sigma}+\alpha\right]
$$

Given that $\frac{L}{1-S}=\frac{\tilde{L}}{1-\tilde{S}}$ and $L>\tilde{L}$, a strict inequality always holds under $\alpha \geq 0$. A similar rationale can be used for AU, CLU and NDU. Under $\alpha<0$, the ranking depends on the condition stated in Proposition 12. 